\title{
Discretizing singular point sources in hyperbolic wave propagation problems
}

\author{
N. Anders Petersson ${ }^{\mathrm{a}, *}$, Ossian O'Reilly ${ }^{\mathrm{b}}$, Björn Sjögreen $^{\mathrm{a}}$, Samuel Bydlon ${ }^{\mathrm{b}}$ \\ ${ }^{a}$ Center for Applied Scientific Computing, Lawrence Livermore National Laboratory, PO Box 808, \\ Livermore CA 94551 \\ ${ }^{b}$ Department of Geophysics, Stanford University, Stanford, CA 94305.
}

\begin{abstract}
We develop high order accurate source discretizations for hyperbolic wave propagation problems in first order formulation that are discretized by finite difference schemes. By studying the Fourier series expansions of the source discretization and the finite difference operator, we derive sufficient conditions for achieving design accuracy in the numerical solution. Only half of the conditions in Fourier space can be satisfied through moment conditions on the source discretization, and we develop smoothness conditions for satisfying the remaining accuracy conditions. The resulting source discretization has compact support in physical space, and is spread over as many grid points as the number of moment and smoothness conditions. In numerical experiments we demonstrate high order of accuracy in the numerical solution of the 1-D advection equation (both in the interior and near a boundary), the 3-D elastic wave equation, and the 3-D linearized Euler equations.
\end{abstract}

Keywords: Singular sources, hyperbolic wave propagation, moment conditions, smoothness conditions, summation by parts

2010 MSC: 65M06, 65M12, 86-08

\section{Introduction}

Singular source terms are used in several areas of computational physics, for example to model sound generation in the atmosphere $[13,7]$, or to model earthquakes in seismic wave simulations $[3,1]$. Singular source terms are also used to model fluid-structure interaction in the immersed boundary method [18], and in applications of the level set method [16, 23, 17], for example to model surface tension along the interface of immiscible fluids. There are also other topics related to the numerical approximations of non-singular source terms, for example, the construction of well-balanced schemes $[5,9,28]$, or the construction of discretizations to mitigate stiff source terms in combustion simulations [29].

${ }^{*}$ Corresponding author

Email addresses: petersson1@llnl.gov (N. Anders Petersson), ooreilly@stanford.edu (Ossian O'Reilly), sjogreen2@llnl.gov (Björn Sjögreen), sbydlon@stanford.edu (Samuel Bydlon)

Preprint submitted to Journal of Computational Physics

April 9, 2016 
Several techniques have been developed for discretizing singular source terms in the context of solving partial differential equations by the finite difference or finite element methods. Waldén [27] developed a theory for predicting the number of moment conditions that must be satisfied in the discrete approximation of a Dirac delta distribution, or its derivatives. He considered finite difference and finite element discretizations of the 1-D Helmholtz equation and proved that design accuracy can be obtained away from the singularity. He also numerically demonstrated 4th order convergence in the solution of a 3-D Helmholtz equation subject to a point source. Petersson and Sjogreen [19] generalized Walden's approach to handle sources near mesh refinement boundaries in a finite difference discretization of the elastic wave equation in second order formulation. Tornberg and Engquist [25] also developed a theory involving moment conditions and analyzed the multi-dimensional case, where singular sources are distributed along a curve or a surface. Using a source discretization that satisfies four moment conditions, they demonstrated 4th order convergence for a 1-D parabolic equation and a 2-D elliptic equation where the source was distributed along a curve. A different source discretization approach, which remains consistent under dilations and works well in conjunction with the level set method, was proposed by Zahedi and Tornberg [30]. Their approach is based on scaling a continuous function that has a rapidly decaying Fourier transform. A tunable tolerance parameter is used to truncate the source discretization to a fixed number of grid points. It is straightforward to satisfy two moment conditions with this approach, but it is unclear if it can be generalized to higher order of accuracy. More recently, Hosseini et al. [11] studied regularizations of Dirac distributions and developed a radially symmetric delta approximation in 2-D. They demonstrated up to 4 th order convergence for Helmholtz equation in 1-D and 2-D, but reported poor convergence for the 1-D advection equation and the 2-D wave equation.

In this paper we develop source discretizations for linear hyperbolic wave propagation problems in first order formulation, where the solution is required to propagate over many wavelengths with minimal dissipation. We therefore focus on high order accurate and non-dissipative finite difference schemes in space that are integrated by explicit high order methods in time. The difference approximations are centered in the interior of the domain, complemented by boundary modifications that satisfy the principle of summation by parts $[24,15]$, and thus are provably stable.

A source discretization that only satisfies moment conditions will in general trigger all modes on the grid. When a centered finite difference scheme is applied to a linear hyperbolic problem, only the well-resolved modes are accurately propagated on the grid. Modes that are poorly resolved are not damped by the scheme and are susceptible to large dispersive errors. Once triggered by the source, these modes can result in large errors in the numerical solution. Hence, for a source discretization to be useful within this class of numerical methods, it must be designed to not trigger any poorly resolved modes.

By studying the Fourier series expansions of the source discretization and the difference operator, we derive sufficient conditions for achieving design accuracy in the numerical solution. Only half of the conditions in Fourier space can be satisfied through moment conditions on the source discretization, and we develop smoothness conditions for satisfying the remaining accuracy conditions. The resulting source discretization has compact support in physical space, and is distributed over as many grid points as the number of moment and smoothness conditions. 
To outline our approach, we consider the 1-D advection equation with a singular point source, represented by the Dirac distribution $\delta$,

$$
\begin{aligned}
u_{t}+u_{x} & =g(t) \delta\left(x-x_{*}\right), \quad-\infty<x<\infty, \quad t>0, \\
u(x, 0) & =0, \quad-\infty<x<\infty .
\end{aligned}
$$

We assume that the source time function $g(t)$ is smooth and $g(0)=0$. The solution of (1) is

$$
u(x, t)= \begin{cases}g\left(t-\left(x-x_{*}\right)\right), & 0 \leq x-x_{*}<t, \\ 0, & \text { otherwise. }\end{cases}
$$

The solution is as smooth as the time function, except along the line $x=x_{*}$ in the $x$ - $t$ plane, where it jumps from 0 to $g(t)$. If $g(t)=0$ for $t>t_{1}$, the jump vanishes after that time and the solution becomes a smooth traveling wave, see Figure 1.

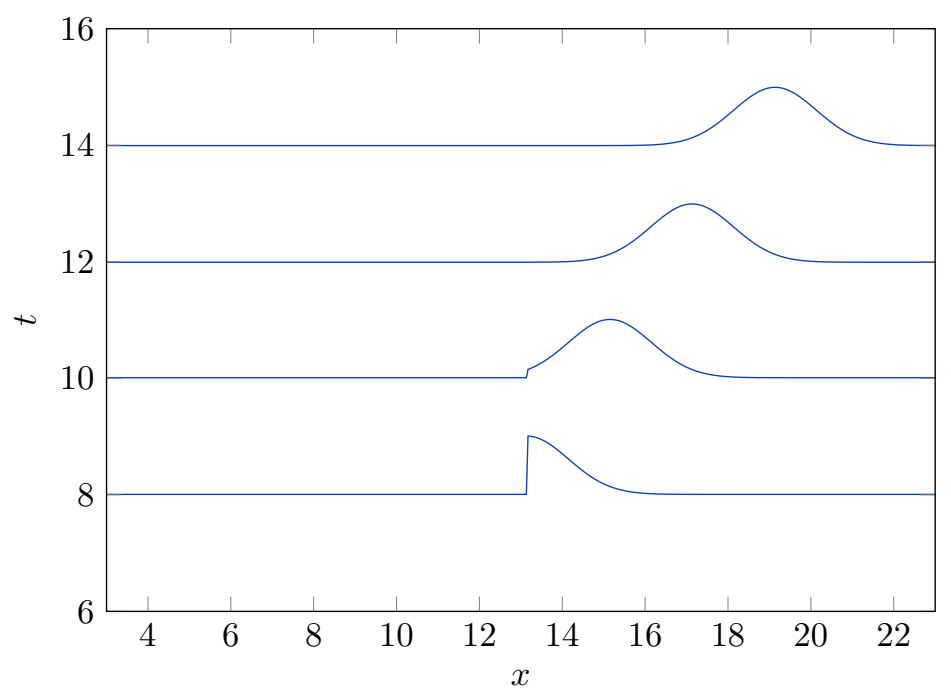

Figure 1: Space-time plot of the analytical solution (2) when the time-function is a Gaussian.

Because the analytical solution is identically zero for $x<x_{*}$ and $x>x_{*}+t$, we can for bounded times replace the unbounded domain in (1) by a sufficiently large periodic domain. In the following we assume that $u(x, t)$ is $L$-periodic in $x$ and take the domain to be $x \in[0, L]$. We also assume that the source location satisfies $0<x_{*} \leq L / 2$. The solution formula (2) is then valid for $0 \leq t \leq L-x_{*}$.

The problem (1) on the periodic domain $x \in[0, L]$ is discretized in space by a finite difference method on a grid $x_{j}=j h, j=0,1, \ldots, N-1$, with constant grid size $h=L / N$. We are interested in the general case where the source is located at an arbitrary location relative to the grid,

$$
x_{*}=x_{k}+\alpha h, \quad k=\left\lfloor x_{*} / h\right\rfloor, \quad 0 \leq \alpha<1 .
$$


The periodic grid function $v_{j}(t) \approx u\left(x_{j}, t\right), v_{j}=v_{j+N}$, satisfies the semi-discrete problem,

$$
\begin{aligned}
\frac{d}{d t} v_{j}(t) & =-D v_{j}+g(t) d_{j}, \quad j=0,1, \ldots, N-1, \quad t>0, \\
v_{j}(0) & =0 .
\end{aligned}
$$

Here, $D$ is a $p$ th order accurate centered finite difference operator, see Appendix A for details, and $d_{j}$ is the discretization of the Dirac delta distribution, which often is written on the form

$$
d_{j}=\delta_{\varepsilon}^{\{M, S\}}\left(x_{j}-x_{*}\right), \quad \delta_{\varepsilon}^{\{M, S\}}(x)=\frac{1}{h} \varphi^{\{M, S\}}\left(\frac{x}{h}\right) .
$$

Here, superscripts $M$ and $S$ correspond to the number of moment and smoothness conditions that $d$ satisfies. These conditions are defined in Section 2.

One of the simplest source discretizations is the narrow hat function, corresponding to a grid function that is non-zero at $d_{k}$ and $d_{k+1}$,

$$
\varphi^{\{2,0\}}(\xi)=\left\{\begin{array}{lr}
1-|\xi|, & |\xi|<1, \\
0, & \text { otherwise. }
\end{array}\right.
$$

Unfortunately, significant amounts of spurious numerical oscillations are triggered if this source discretization is combined with a centered finite difference approximation in space, and integrated by the explicit 4th order Runge-Kutta (RK-4) scheme in time, see Figure 2 (the fact that the spurious waves travel in the opposite direction of the physical waves is a consequence of the centered difference approximation, see e.g. [26]).

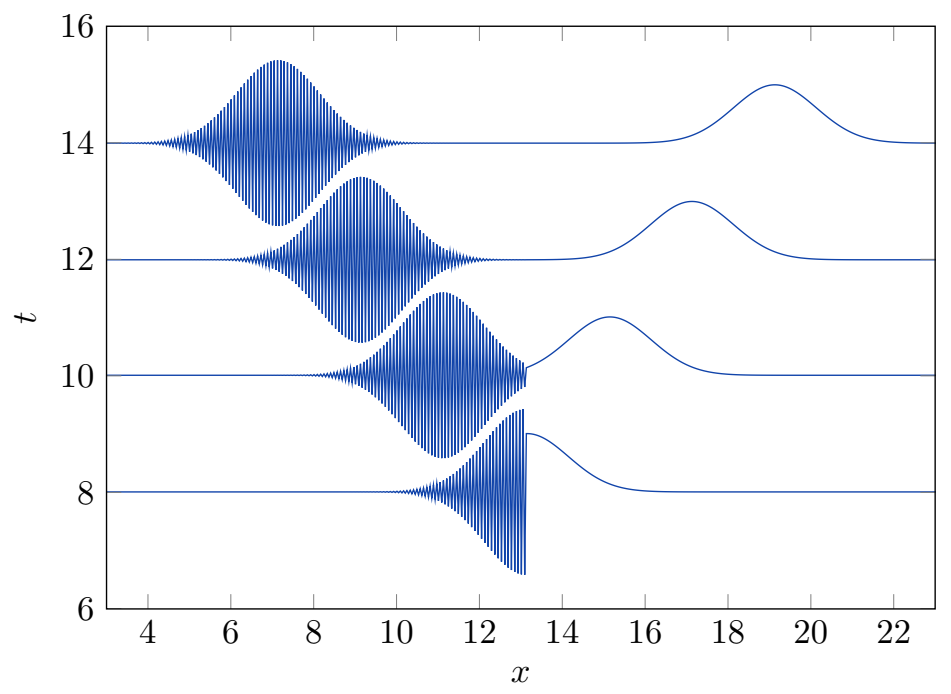

Figure 2: Space-time plot of the numerical solution of (4) with a 2nd order centered finite difference scheme and the source discretization $\delta_{\varepsilon}^{\{2,0\}}$, which satisfies two moment conditions but no smoothness conditions.

To control the spurious oscillations we propose a modified source discretization formula, which satisfies a number of moment and smoothness conditions. The moment 
conditions that are necessary for achieving $p$ th order accuracy are documented and analyzed in the literature $[27,25]$. To reduce "checkerboard" instabilities in the immersed boundary method, Peskin [18] enforces the "even-odd" condition on the source discretization. This condition is also used in the analysis by Mori [14]. The "even-odd" condition is equivalent to $d_{j}$ being orthogonal to the Nyquist mode $(-1)^{j}$. We call this the fundamental smoothness condition. In Section 2 we introduce higher order smoothness conditions that make $d_{j}$ orthogonal to the grid functions $(-1)^{j}\left(x_{j}\right)^{q}$, for $q=1,2, \ldots, S-1$. We also develop a theory for $p$ th order centered finite difference approximations of the advection equation, showing that $p$ moment and $p$ smoothness conditions are required for full accuracy of the approximation.

For 2nd order accuracy, our approach leads to a source discretization constructed from the wide hat-function,

$$
\varphi^{\{2,2\}}(\xi)= \begin{cases}\frac{1}{4}(2-|\xi|), & |\xi|<2, \\ 0, & \text { otherwise }\end{cases}
$$

With this source discretization the numerical solution is free from spurious oscillations, see Figure 3, and it can be verified that the numerical solution is 2 nd order accurate once the discontinuity has vanished. We emphasize that the differences between the results in Figures 2 and 3 are solely due to the source discretization. All other aspects of the numerical modeling are identical.

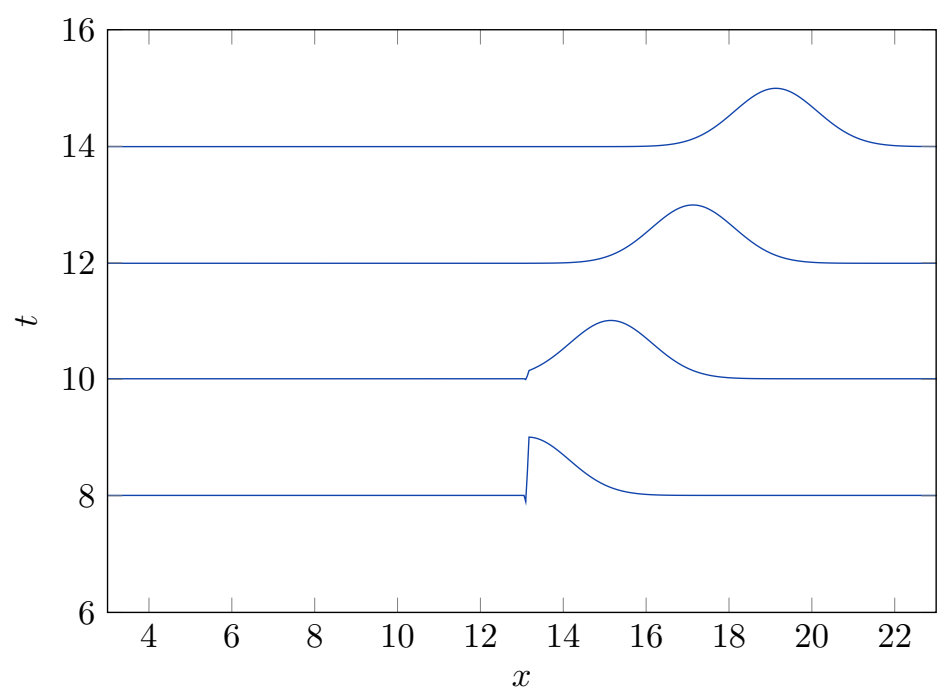

Figure 3: Numerical solution of (4) with a 2nd order finite difference scheme using the source discretization $\delta_{\varepsilon}^{\{2,2\}}$, which satisfies two moment conditions and two smoothness conditions.

To get 4th order accuracy our source discretization results in the piecewise cubic 
polynomial,

$$
\varphi^{\{4,4\}}(\xi)= \begin{cases}\frac{1}{32}\left(16-4|\xi|-4|\xi|^{2}+|\xi|^{3}\right), & |\xi|<2 \\ \frac{1}{96}\left(48-44|\xi|+12|\xi|^{2}-|\xi|^{3}\right), & |\xi| \in[2,4) \\ 0, & \text { otherwise }\end{cases}
$$

For 6th order accuracy, we arrive at the piecewise quintic polynomial,

$$
\varphi^{\{6,6\}}(\xi)= \begin{cases}\frac{1}{2}-\frac{1}{12}|\xi|-\frac{5}{32}|\xi|^{2}+\frac{5}{192}|\xi|^{3}+\frac{1}{128}|\xi|^{4}-\frac{1}{768}|\xi|^{5}, & |\xi|<2, \\ \frac{1}{2}-\frac{13}{48}|\xi|-\frac{5}{64}|\xi|^{2}+\frac{25}{384}|\xi|^{3}-\frac{3}{256}|\xi|^{4}+\frac{1}{1536}|\xi|^{5}, & |\xi| \in[2,4), \\ \frac{1}{2}-\frac{137}{240}|\xi|+\frac{15}{64}|\xi|^{2}-\frac{17}{384}|\xi|^{3}+\frac{1}{256}|\xi|^{4}-\frac{1}{7680}|\xi|^{5}, & |\xi| \in[4,6), \\ 0, & \text { otherwise }\end{cases}
$$

which satisfies six moment and six smoothness conditions. The source discretization functions (7), (8), and (9) are plotted in Figure 4.

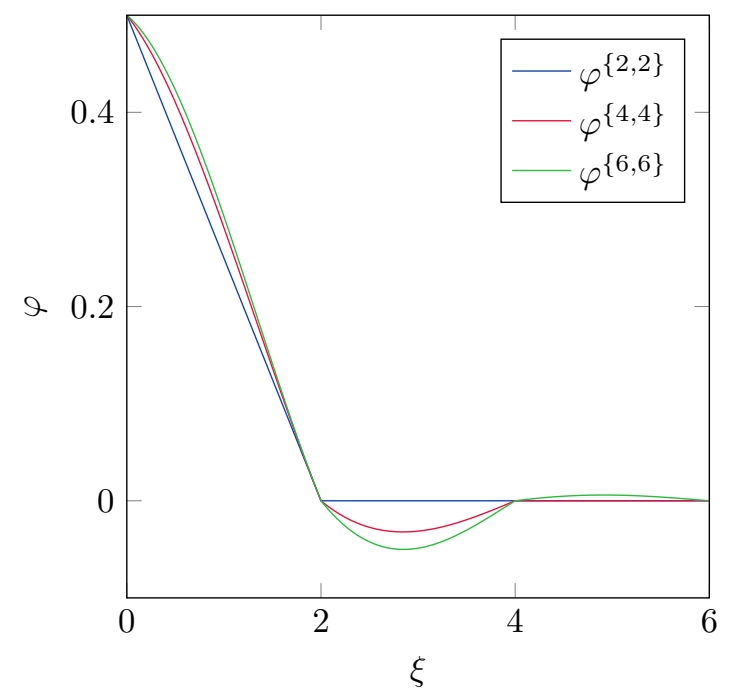

Figure 4: The source discretization functions used for 2nd, 4th, and 6th order accuracy.

The remainder of the paper is organized as follows. The moment and smoothness conditions are described in Section 2. In Section 3 we prove that an (almost) $p$ th order accurate numerical solution of (1) can be obtained from a $p$ th order accurate centered finite difference scheme, if the time function $g(t)$ is sufficiently smooth and the source discretization satisfies $p$ moment conditions as well as $p$ smoothness conditions. In Section 4 , we numerically solve the advection equation (1) using centered difference schemes of orders $p=2,4,6$, and evaluate how the convergence rate depends on the number of smoothness conditions. These experiments confirm the theoretical prediction that a source discretization with $M=S=p$ is necessary for $p$ 'th order accuracy. The remainder of Section 4 introduces two generalizations. In Section 4.1 we solve the advection equation (1) in curvilinear coordinates and numerically verify the $p^{\prime}$ th order convergence 
rate for $p=2,4$, and 6 . In Section 4.2 we numerically derive source discretizations for sources in the vicinity of physical boundaries, in conjunction with a finite difference scheme that satisfies the principle of summation by parts. Section 5 describes two applications of our technique to three-dimensional wave propagation problems, where the source is approximated by a Cartesian product of one-dimensional source discretizations. Conclusions are given in Section 6.

\section{Moment and smoothness conditions}

We start by defining a discrete scalar product and norm for complex-valued grid functions $u_{j}$ and $v_{j}$,

$$
(u, v)_{h}:=h \sum_{j=0}^{N-1} \bar{u}_{j} v_{j}, \quad\|u\|_{h}=\sqrt{(u, u)_{h}},
$$

which is an approximation of $\int_{0}^{L} \bar{u} v d x$. Let the source discretization be represented by the real-valued grid function $d_{j}$ and recall the definition of the Dirac distribution, i.e., $\int \phi(x) \delta\left(x-x_{*}\right) d x=\phi\left(x_{*}\right)$. To ensure that $d_{j}$ is a consistent and accurate discretization of $\delta\left(x-x_{*}\right)$, we enforce $M>0$ moment conditions ${ }^{1}[27,18,25]$,

$$
\left(d, \phi^{(m)}\right)_{h}=\phi^{(m)}\left(x_{*}\right), \quad \phi^{(m)}(x)=x^{m}, \quad m=0,1, \ldots, M-1,
$$

where we define $x^{0}=1$ for all $x$.

As demonstrated by our initial numerical example, the source discretization can trigger spurious modes in the numerical solution. To analyze the spurious modes, let $\psi$ be a function that is not resolved on the grid (such as the Nyquist mode). Consider the scalar product between $\psi$ and (4)

$$
\frac{d}{d t}(\psi, v)_{h}=-(\psi, D v)_{h}+g(t)(\psi, d)_{h} .
$$

This equation governs the evolution of $(\psi, v)_{h}$, which is a spurious component of the numerical solution. If $(\psi, D v)_{h}=0$, we can only prevent $(\psi, v)_{h}$ from growing in time by enforcing the condition $(\psi, d)_{h}=0$ on the source discretization. Assuming that the number of grid points in the periodic domain $(N)$ is even, it is straightforward to construct an $N$-periodic grid function $\psi$ for which $(\psi, D v)_{h}=0$. We have,

$$
(\psi, D v)_{h}=h \psi^{T} D v=h\left(D^{T} \psi\right)^{T} v,
$$

where $D^{T}$ is the transpose of the difference operator $D$ written in matrix form. Thus, $(\psi, D v)_{h}=0$ for all $v$, if the vector $\psi$ satisfies $D^{T} \psi=0$, i.e. $\psi$ is a null vector of $D^{T}$. Because $D$ is skew-symmetric, $D^{T} \psi=-D \psi$. Furthermore, because $D$ is a centered difference approximation, $D \psi^{(0)}=0$ for $\psi_{j}^{(0)}=(-1)^{j}$. Thus, the spurious component of

\footnotetext{
${ }^{1}$ In this context, $\phi^{(m)}(x)$ does not denote the $m$ 'th derivative of $\phi$.
} 
the numerical solution, $\left(\psi^{(0)}, v\right)_{h}$, is zero for $t>0$, if it is initially zero, and the source discretization satisfies the smoothness condition

$$
\left(\psi^{(0)}, d\right)_{h}=0 .
$$

A periodic grid function $w_{j}=w_{j+N}$ can be analyzed through its Fourier series expansion,

$$
w_{j}=\sum_{q} \widehat{w}_{q} e^{i 2 \pi q x_{j} / L}, \quad x_{j}=j h, \quad h=\frac{L}{N} .
$$

When $N$ is even, the highest mode on the grid corresponds to the Nyquist wave number $q=\frac{N}{2}$. Because $N x_{j} / L=j$,

$$
e^{i 2 \pi \frac{N}{2} x_{j} / L}=\left(e^{i \pi}\right)^{j}=(-1)^{j}=\psi^{(0)} .
$$

Thus, (13) implies that $d$ is orthogonal to the Nyquist mode. To further suppress spurious oscillations in the numerical solution, a natural generalization is to require the source discretization to be orthogonal to the second most oscillatory mode on the grid, corresponding to $q=\frac{N}{2}-1$,

$$
e^{i 2 \pi\left(\frac{N}{2}-1\right) x_{j} / L}=(-1)^{j} e^{-i 2 \pi x_{j} / L}=(-1)^{j}\left(1-\frac{2 \pi i}{L} x_{j}+\frac{1}{2}\left(\frac{2 \pi i}{L} x_{j}\right)^{2}+\ldots\right) .
$$

By retaining the first $S$ terms in the Taylor series expansion, we arrive at our proposed smoothness conditions,

$$
\left(d, \psi^{(s)}\right)_{h}=0, \quad \psi_{j}^{(s)}=(-1)^{j}\left(x_{j}\right)^{s}, \quad s=0,1, \ldots, S-1 .
$$

These conditions are well-defined for source discretizations of periodic and non-periodic problems, as long as the source is located away from the end points. Section 4.2 discusses generalizations of the smoothness conditions when the source is located near a boundary.

We construct the source discretization to be non-zero at $M+S$ grid points centered around $x_{*}$, and zero everywhere else. The non-zero entries of $d_{j}$ are determined by satisfying the $M+S$ linear equations of (11) and (14). Given $\alpha$, where $x_{*}=x_{k}+\alpha h$, this system can be solved numerically or symbolically.

Figure 5 (left) shows the magnitude of the Fourier coefficients, $\left|\widehat{d}_{q}\right|$, corresponding to $M=2$ and $S=\ell$ for the cases $\ell=0,1,2$, plotted as function of the scaled wave number $\omega_{q} h / \pi$, where $\omega_{q}=2 \pi q / L$. Figure 5 (right) shows the corresponding Fourier spectrum for source discretizations that satisfy six moment conditions and different numbers of smoothness conditions. The effect of increasing the number of moment conditions from two to six is that $\left|\widehat{d}_{q}\right|$ remains closer to unity further away from $\omega_{q} h=0$. The result of increasing the number of smoothness conditions is to make the spectrum decay faster towards zero as $\left|\omega_{q} h\right| \rightarrow \pi$. The Fourier spectrum of a source discretization with four moment conditions shows an intermediate behavior (not shown to conserve space).

The $M+S$ linear equations of (11) and (14) can be solved symbolically by a computer algebra program such as Maple, illustrating that each non-zero coefficient $d_{j}$ is a polynomial in $\alpha$ of order $M-1$. Knowing $d_{j}(\alpha)$ is sufficient for implementing the source discretization. These polynomials can also be translated to the piecewise polynomial form of $\varphi^{\{M, S\}}(\xi)$ by the substitution $\xi=j-k-\alpha$. For $M=S=2,4$, and 6 , this technique leads to the formulae (7), (8), and (9), respectively. 

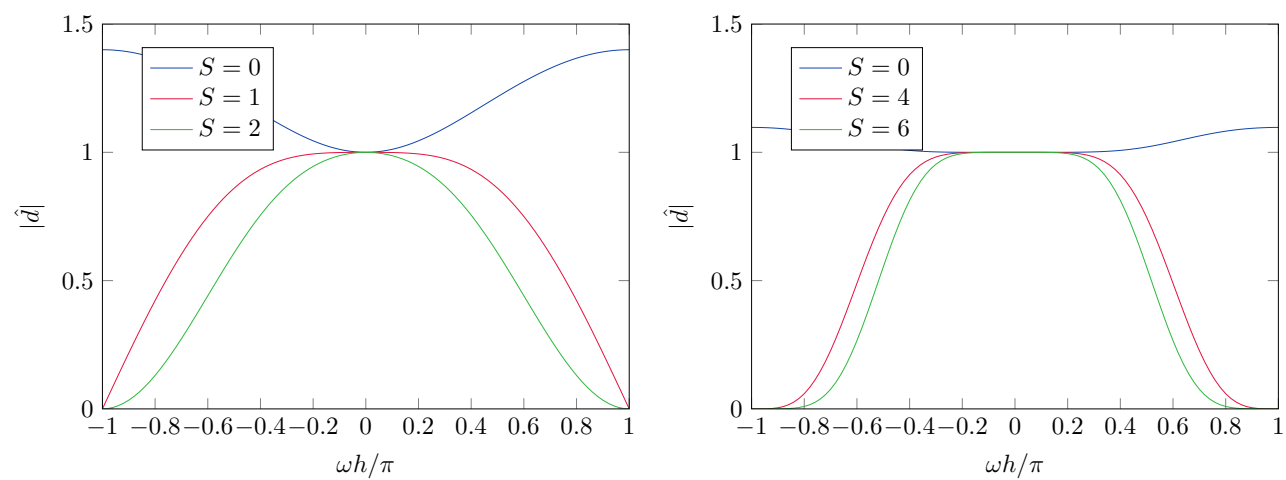

Figure 5: The amplitude of the Fourier coefficients of source discretizations with $M=2$ moment conditions and $S=0,1,2$ smoothness conditions (left), as well as $M=6$ moment conditions and $S=0,4,6$ smoothness conditions (right). Here $\alpha=0.2$.

\section{Accuracy analysis}

We consider spatially periodic solutions of (1) on the domain $0 \leq x<2 \pi$, i.e. assume that the independent variables have been scaled such that $L=2 \pi$. To simplify the presentation we assume that the number of grid points in the spatial discretization, $N$, is odd such that $R:=(N-1) / 2$ is an integer. However, this is no restriction and our theory also holds when $N$ is even. We further assume that the source time function $g(t)$ is smooth and satisfies $g(0)=0$ and $g(t)=0$ for $t>t_{1}$, where $t_{1} \leq 2 \pi-x_{*}$ is some given time. To derive an error estimate, we also need to assume that a sufficient number of derivatives (to be specified below) of $g$ are zero at $t=0$.

The Fourier series expansion of a continuous $2 \pi$-periodic function, $w(x)$, is defined by

$$
w(x)=\sum_{\omega=-\infty}^{\infty} \widehat{w}_{\omega} e^{i \omega x}, \quad \widehat{w}_{\omega}=\frac{1}{2 \pi} \int_{0}^{2 \pi} w(x) e^{-i \omega x} d x, \omega=0, \pm 1, \ldots
$$

Inserting the Fourier expansion into the continuous problem (1) leads to the system of decoupled ordinary differential equations,

$$
\frac{d}{d t} \widehat{u}_{\omega}+i \omega \widehat{u}_{\omega}=\frac{g(t)}{2 \pi} e^{-i \omega x_{*}}, \quad t \geq 0, \omega=0, \pm 1, \ldots,
$$

subject to the initial condition $\widehat{u}_{\omega}(0)=0$. The continuous problem (16) is solved by

$$
\widehat{u}_{\omega}(t)=\frac{1}{2 \pi} \int_{0}^{t} g(\tau) e^{i \omega\left(\tau-t-x_{*}\right)} d \tau, \quad \omega=0, \pm 1, \ldots
$$

The Fourier series expansion of the grid function $v_{j}$ is defined by

$$
v_{j}=\sum_{\omega=-R}^{R} \widehat{v}_{\omega} s_{j}^{(\omega)}, \quad \widehat{v}_{\omega}=\frac{1}{2 \pi}\left(s^{(\omega)}, v\right)_{h}, \quad s^{(\omega)}=e^{i \omega x_{j}}, \quad R=\frac{N-1}{2} .
$$

The basis functions $s^{(\omega)}$ are mutually orthogonal with respect to the scalar product (10), see e.g. [10] (note that we use the hat $\left(\widehat{v}_{\omega}\right)$ to denote the Fourier transform of both a 
continuous and a discrete (grid) function). The semi-discrete problem (4) can also be solved by Fourier expansion,

$$
\widehat{v}_{\omega}(t)=\int_{0}^{t} g(\tau) e^{i \omega P(\omega h)(\tau-t)} \widehat{d}_{\omega} d \tau, \quad \omega=0, \pm 1, \ldots, \pm R .
$$

Since $h=2 \pi / N$, we have $-\pi<\omega h<\pi$. The function $P(\omega h)$ is defined through the Fourier symbol of the finite difference operator $D$,

$$
\widehat{D}(\omega, h)=i \omega P(\omega h) .
$$

For example, the 2 nd order accurate formula $D^{(2)} u_{j}=\left(u_{j+1}-u_{j-1}\right) / 2 h$ has the symbol

$$
\widehat{D}^{(2)}(\omega, h)=i \frac{\sin (\omega h)}{h}=i \omega \frac{\sin (\omega h)}{\omega h}, \quad P^{(2)}(\omega h)=\frac{\sin (\omega h)}{\omega h} .
$$

The symbols of the centered operators for orders $p=2,4,6$ are given in Appendix A. In general, for even orders of accuracy $p \geq 2$, the symbol is of the form (see [10])

$$
P^{(p)}(\omega h)=\frac{\sin (\omega h)}{\omega h}\left(1+\sum_{\nu=1}^{p / 2-1} \alpha_{\nu} \sin ^{2 \nu}(\omega h / 2)\right), \quad \alpha_{\nu}>0, \quad p=2,4,6, \ldots
$$

\section{In the following we suppress the superscript $(p)$ on the operator $P$.}

The following lemma states two essential properties of centered finite difference operators.

Lemma 1. Let $P(\omega h)$ be the function defined by (20), associated with the Fourier symbol of a pth order accurate centered finite difference operator, where $p \geq 2$ is even. There is a cutoff frequency $\xi_{c}>0$ such that

$$
|P(\omega h)-1| \leq c_{1}|\omega h|^{p}, \quad|\omega h| \leq \xi_{c},
$$

and furthermore,

$$
|P(\omega h)| \geq c_{2}(\pi-|\omega h|), \quad|\omega h| \leq \pi,
$$

for some constants $c_{1}$ and $c_{2}$.

Proof. For small $\omega h$, the $p$ th order accuracy implies that

$$
P(\omega h)=1+\mathcal{O}\left((\omega h)^{p}\right),
$$

see [10], which proves (22). Because the expression inside the parenthesis in (21) is positive and greater than unity, the inequality

$$
\sin x \geq \frac{x}{\pi}(\pi-x), \quad 0 \leq x \leq \pi
$$

gives the estimate

$$
|P(\omega h)| \geq c_{2}(\pi-|\omega h|), \quad|\omega h| \leq \pi .
$$

This completes the proof of Lemma 1. 
The lowest order moments condition, i.e., (11) for $M=1$, gives

$$
(d, 1)_{h}=h \sum_{j=0}^{N-1} d_{j}=1 .
$$

We construct the source discretization to be non-zero at $M+S$ grid points in the vicinity of $x_{*}$, and zero everywhere else. This means that the non-zero coefficients must scale as $d_{j}=\mathcal{O}(1 / h)$. The Fourier coefficients of the source discretization are defined by

$$
\widehat{d}_{\omega}=\frac{1}{2 \pi}\left(s^{(\omega)}, d\right)_{h}=\frac{h}{2 \pi} \sum_{j=0}^{N-1} e^{-i \omega h j} d_{j}, \quad \omega=0, \pm 1, \ldots, \pm R .
$$

Because $d_{j}=\mathcal{O}(1 / h)$, we conclude that $\widehat{d}_{\omega}$ is a function of $\omega h$.

The next lemma establishes some properties of the source discretization.

Lemma 2. Let $p>0$ be a natural number and let the source discretization satisfy the moment conditions (11) for $M=p$ and the smoothness conditions (14) for $S=p$. Then there is a cutoff frequency $\xi_{c}>0$ such that

$$
\left|\widehat{d}_{\omega}-e^{-i \omega x_{*}} / 2 \pi\right| \leq c_{3}|\omega h|^{p}, \quad|\omega h| \leq \xi_{c},
$$

and furthermore,

$$
\left|\widehat{d}_{\omega}\right| \leq c_{4}(\pi-|\omega h|)^{p}, \quad|\omega h| \leq \pi,
$$

for some constants $c_{3}$, and $c_{4}$.

Proof. See Appendix B.

\subsection{Error estimate}

By Fourier interpolation, we can define a continuous function $\operatorname{Int}_{N} v(x, t)$ that satisfies

$$
\operatorname{Int}_{N} v\left(x_{j}, t\right)=v_{j}(t), \quad j=0,1, \ldots, N-1 .
$$

The coefficients of its (continuous) Fourier expansion, defined by (15), are zero for $|\omega h|>$ $\pi$. For $|\omega h|<\pi$, they are identical to the coefficients of the (discrete) Fourier expansion of the grid function $v_{j}(t)$, defined by (18). We refer to [10] for a proof of this and other properties of Fourier interpolation.

We shall estimate the solution error, $e(x, t):=u(x, t)-\operatorname{Int}_{N} v(x, t)$, through Parseval's relation,

$$
\|e(\cdot, t)\|^{2}=2 \pi \sum_{\omega=-\infty}^{\infty}\left|\widehat{e}_{\omega}(t)\right|^{2}=2 \pi \sum_{\omega=-R}^{R}\left|\widehat{e}_{\omega}(t)\right|^{2}+2 \pi \sum_{|\omega|=R+1}^{\infty}\left|\widehat{u}_{\omega}(t)\right|^{2},
$$

for some fixed time $t>t_{1}$. Here, $\|e\|$ is the continuous $L_{2}$-norm of $e$. In the 2nd sum, the Fourier coefficients of the error equal $\widehat{u}_{\omega}$ because the Fourier coefficients of $\operatorname{Int}_{N} v(x, t)$ are zero for $|\omega| \geq R+1$. If the time-function of the continuous problem, $g(t)$, has at least 
$p+1$ continuous derivatives, we can prove by $p+1$ partial integrations of the solution formula (17) that

$$
\left|\widehat{u}_{\omega}(t)\right| \leq \frac{C}{|\omega|^{p+1}}, \quad t>t_{1} .
$$

This implies that

$$
\sum_{|\omega|=R+1}^{\infty}\left|\widehat{u}_{\omega}\right|^{2} \leq C \sum_{|\omega|=R+1}^{\infty} \frac{h^{2 p}}{|\omega|^{2}|\omega h|^{2 p}} \leq C h^{2 p},
$$

because $1 /|\omega h|<1 / \pi$ and $\sum \frac{1}{|\omega|^{2}}$ is a convergent sum (here and below we use $C$ to denote a positive constant that can be different between different invocations). We conclude that the 2 nd sum on the right hand side of (27) does not influence the order of convergence of the error up to and including order $p$. Thus, the error in the solution is controlled by the Fourier coefficients

$$
\widehat{e}_{\omega}(t)=\widehat{u}_{\omega}(t)-\widehat{v}_{\omega}(t), \quad \omega=0, \pm 1, \ldots, \pm R .
$$

Our main result is stated in Theorem 1.

Theorem 1. Let the centered difference operator $D$ be pth order accurate and let the source discretization satisfy $M=p$ moment conditions and $S=p$ smoothness conditions, where $p \geq 2$ is even. Furthermore, let $g(t)$ have $p+m$ continuous derivatives, and be zero for $t \leq 0$ and $t \geq t_{1}$. Then the error in the solution of (4) satisfies the estimate

$$
\|e\| \leq C h^{p-\frac{1}{2 m}}, \quad m \geq 2,
$$

for any fixed time $t>t_{1}$.

Remark 1. Numerical experiments indicate that the error converges as $\|e\|=C h^{p}$ when $g(t)$ has $p+2$ continuous derivatives and $M=S=p$. This observation either indicates that the above estimate is not sharp, or that it is numerically difficult to distinguish between the convergence rates $h^{p}$ and $h^{p-1 / 4}$.

Our proof of Theorem 1 relies on Lemma 3.

Lemma 3. Let $\widehat{e}_{\omega}$ be the Fourier coefficients of the error in the numerical solution obtained with a finite difference scheme and a source discretization as specified in Theorem 1. Then, for some constant $\eta>0$,

$$
\begin{array}{rlrl}
\widehat{e}_{\omega} & =0, & \omega & =0, \\
\left|\widehat{e}_{\omega}\right| & \leq C_{1} h^{p} /|\omega|, & h \leq|\omega h|<\pi-\eta h^{\frac{m-1}{m}}, \\
\left|\widehat{e}_{\omega}\right| & \leq C_{2} h^{p}, & \pi-\eta h^{\frac{m-1}{m}} \leq|\omega h|<\pi .
\end{array}
$$

Proof. See Appendix C.

To prove Theorem 1 we introduce the natural number $R_{1}$ such that $R_{1}+1 \leq R=\frac{\pi}{h}-\frac{1}{2}$,

$$
R_{1}<\frac{\pi}{h}-\eta h^{-1 / m} \leq R_{1}+1, \quad\left|R_{1} h\right|<\pi-\eta h^{(m-1) / m},
$$


and split the sum in Parseval's relation (27) such that

$$
\|e(t)\|^{2}=2 \pi\left[\left|\widehat{e}_{0}\right|^{2}+\sum_{|\omega|=1}^{R_{1}}\left|\widehat{e}_{\omega}\right|^{2}+\sum_{|\omega|=R_{1}+1}^{R}\left|\widehat{e}_{\omega}\right|^{2}\right]
$$

Note that the 2 nd sum has $2 N_{2}$ terms, where

$$
N_{2}=R-R_{1}<\left(\frac{\pi}{h}-\frac{1}{2}\right)-\left(\frac{\pi}{h}-\eta h^{-1 / m}-1\right)=\eta h^{-1 / m}+\frac{1}{2} .
$$

The two sums in (33) correspond to cases (31) and (32) of Lemma 3,

$$
\|e(t)\|^{2} \leq 2 \pi C_{1}^{2} h^{2 p} \sum_{|\omega|=1}^{R_{1}} \frac{1}{|\omega|^{2}}+2 \pi C_{2}^{2} \sum_{|\omega|=R_{1}+1}^{R} h^{2 p} \leq C_{3} h^{2 p}+C_{4} h^{2 p} h^{-1 / m} \leq C h^{2 p-1 / m} .
$$

Here we used that $\widehat{e}_{0}=0$, and $\sum \frac{1}{|\omega|^{2}}$ is convergent, so that the first sum can be bounded independently of $R_{1}$ (and $h$ ). Furthermore, we used that the number of terms in the second sum, $2 N_{2}=\mathcal{O}\left(h^{-1 / m}\right)$. The estimate (29) follows by taking the square root of $(34)$.

\section{Computational experiments in 1-D}

We begin by investigating the error in the numerical solution of the model problem (1), discretized in space according to (4) and integrated in time by the classical explicit RK-4 scheme.

In our numerical experiments we take the domain size to be $L=40$, locate the source at $x_{*}=10+\pi$, and use the Gaussian time function

$$
g(t)=\exp \left(-\frac{\left(t-t_{0}\right)^{2}}{2}\right), \quad t_{0}=8
$$

The center time, $t_{0}=8$, is chosen to ensure that the source time function is sufficiently small at $t=0$ to avoid any noticeable artifacts in double precision arithmetic. The exact solution, given by (2), consists of a Gaussian wave packet traveling to the right, see Figure 1. Formally, the analytical solution remains discontinuous at the source location for as long as $g(t)>0$, but it can for numerical purposes be considered smooth for $t \geq 2 t_{0}=16$.

We define the error in the numerical solution as the difference between the numerical and the analytical solutions

$$
e_{j}(t)=u\left(x_{j}, t\right)-v_{j}(t) .
$$

We integrate the numerical solution until the time $t=T:=20$, at which point we measure the error in the discrete $l_{2}$-norm (10). This time is chosen to make the solution smooth and periodic in space, i.e., $T>2 t_{0}=16$ and $T<L-x_{*}=30-\pi$, respectively.

We use a $p$ th order accurate finite difference discretization for $p=2,4,6$, and $N=$ $70 \times 2^{k-1}$ grid points for $k=1,2, \ldots, 7$. In other words, the grid spacing is reduced by a factor of two with each level of grid refinement. We use the constant time step $\Delta t=h / 2$ 


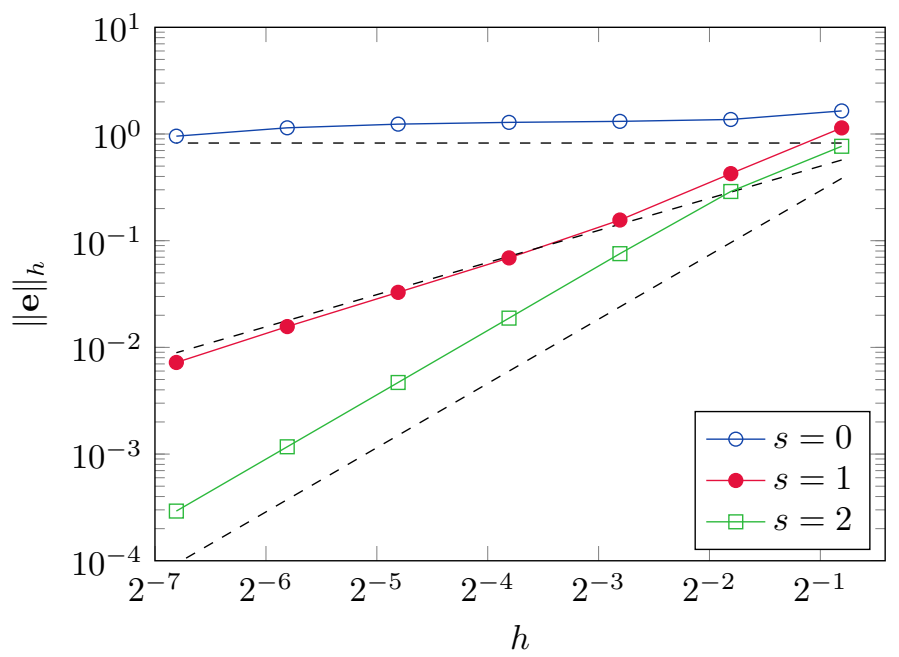

Figure 6: The error in the numerical solution with a 2nd order finite difference scheme and a source discretization with $M=2$ moment condition and $S=0,1,2$ smoothness conditions. The dashed lines show $\|e\|_{h} \sim h^{q}$, for $q=0,1,2$, respectively.

for the 2 nd and 4 th order finite difference schemes. For the 6 th order case, the time step is reduced to $\Delta t=h^{3 / 2} / 2$ to make the temporal error proportional to $O\left(h^{6}\right)$.

We construct the source discretization to satisfy $M=p$ moment conditions, and we vary the number of smoothness conditions according to $S=0,1, \ldots, p$. The results are presented in Figures 6 - 8. We conclude that the numerical solution becomes $p$ th order accurate when the source discretization satisfies $M=p$ moment conditions and $S=p$ smoothness conditions.

\subsection{Curvilinear coordinates}

In this section we demonstrate how to apply our source discretization on a nonuniform grid using coordinate transformations. Let the coordinate transformation

$$
x=X(\widetilde{x}),
$$

be a smooth one-to-one mapping from the unit interval in parameter space $\widetilde{x} \in[0,1]$ to the interval $x \in[0, L]$ in physical space. Applying the coordinate transformation to the model problem (1) leads to

$$
\widetilde{u}_{t}+\frac{1}{X_{\widetilde{x}}} \widetilde{u}_{\widetilde{x}}=g(t) \delta\left(X(\widetilde{x})-X\left(\widetilde{x}_{*}\right)\right), \quad 0 \leq \widetilde{x} \leq 1, \quad t \geq 0,
$$

where $\widetilde{u}(\widetilde{x}, t)=u(X(\widetilde{x}), t)$. Here $\widetilde{x}_{*}$ is the parameter coordinate of the source location, determined by solving $x_{*}=X\left(\widetilde{x}_{*}\right)$ for $\widetilde{x}_{*}$. The mapping is assumed to be non-singular and the Jacobian of the mapping satisfies $J(\widetilde{x})=X_{\widetilde{x}}(\widetilde{x})$. The Dirac distribution transforms according to

$$
\delta\left(X(\widetilde{x})-X\left(\widetilde{x}_{*}\right)\right)=\frac{\delta\left(\widetilde{x}-\widetilde{x}_{*}\right)}{J(\widetilde{x})}, \quad \int \phi(\widetilde{x}) \delta\left(\widetilde{x}-\widetilde{x}_{*}\right) d \widetilde{x}=\phi\left(\widetilde{x}_{*}\right),
$$




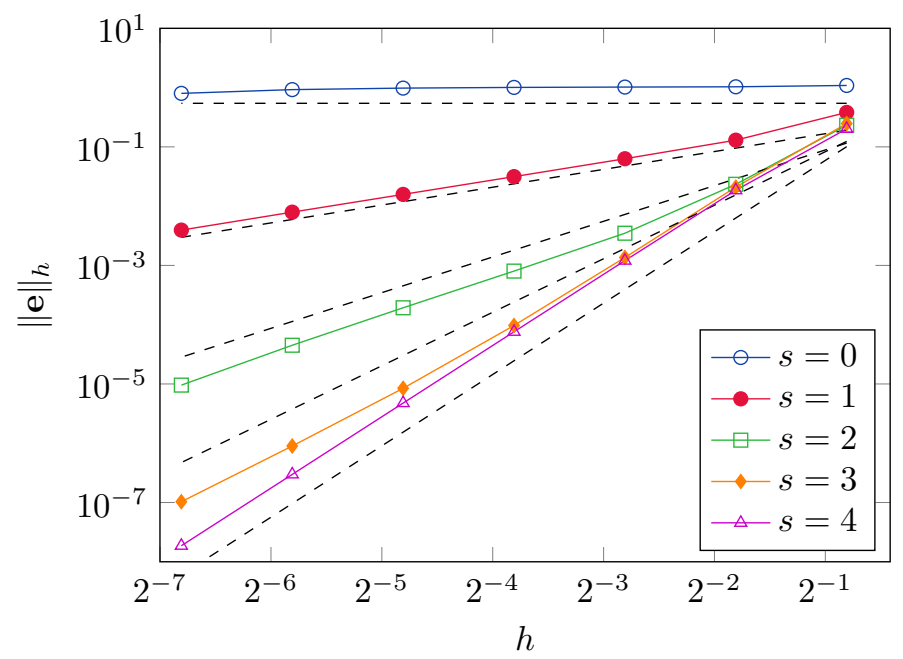

Figure 7: The error in the numerical solution with a 4th order finite difference scheme and a source discretization with $M=4$ moment condition and $S=0,1,2,3,4$ smoothness conditions. The dashed lines show $\|e\|_{h} \sim h^{q}$, for $q=0,1,2,3,4$, respectively.

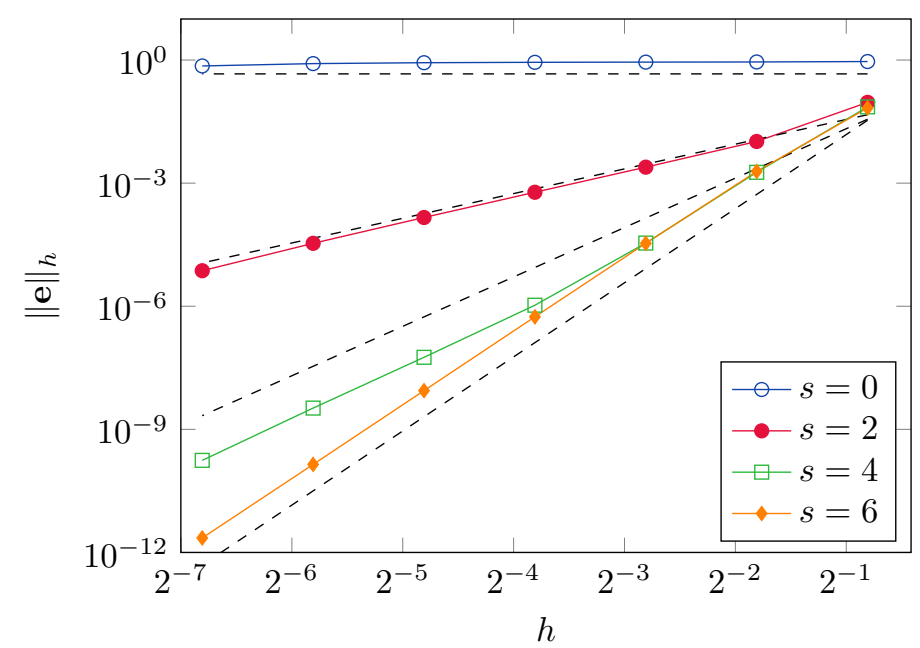

Figure 8: The error in the numerical solution with a 6th order finite difference scheme and a source discretization with $M=6$ moment condition and $S=0,2,4,6$ smoothness conditions. The dashed lines show $\|e\|_{h} \sim h^{q}$, for $q=0,2,4,6$, respectively. 
where $\phi(\tilde{x})$ is a smooth function. Thus, the model problem (37) can be written

$$
\widetilde{u}_{t}+\frac{1}{J(\widetilde{x})} \widetilde{u}_{\widetilde{x}}=g(t) \frac{\delta\left(\widetilde{x}-\widetilde{x}_{*}\right)}{J(\widetilde{x})}, \quad 0 \leq \widetilde{x} \leq 1, t \geq 0 .
$$

We introduce a uniform grid in parameter space, $\widetilde{x}_{j}=j \widetilde{h}, j=0, \ldots, N-1$ with grid size $\widetilde{h}=1 / N$. Let the grid function $\widetilde{v}_{j}(t)$ approximate $\widetilde{u}\left(\widetilde{x}_{j}, t\right)$, and discretize (38) according to

$$
\frac{d}{d t} \widetilde{v}_{j}+\frac{1}{J_{j}} D^{(\widetilde{x})} \widetilde{v}_{j}=g(t) \frac{\delta_{\varepsilon}\left(\widetilde{x}_{j}-\widetilde{x}_{*}\right)}{J_{j}}, \quad j=0,1, \ldots, N-1 .
$$

Since the grid is uniform in parameter space, $D^{(\widetilde{x})}$ is the same $p$ th order accurate centered finite difference operator as $D$ (with grid size $\widetilde{h}$ ). The source discretization follows by replacing $h$ by $\widetilde{h}$ in $(5)$, i.e., $\delta_{\varepsilon}(\widetilde{x})=\frac{1}{\tilde{h}} \varphi(\tilde{x} / \tilde{h})$.

To test the source discretization on a non-uniform grid we introduce the mapping

$$
X(\widetilde{x})=\frac{L}{2}\left(1+\widetilde{x} \sin \left(\frac{\pi}{2} \widetilde{x}\right)\right) \widetilde{x}
$$

and compute

$$
J:=X_{\widetilde{x}}=\frac{L}{2}\left(1+2 \widetilde{x} \sin \left(\frac{\pi}{2} \widetilde{x}\right)+\frac{\pi}{2} \widetilde{x}^{2} \cos \left(\frac{\pi}{2} \widetilde{x}\right)\right) .
$$

It is straightforward to verify that $X(\widetilde{x})$ is non-singular for $0 \leq \widetilde{x} \leq 1$.

We perform a convergence study by choosing $L=40, \widetilde{\widetilde{x}}_{*}=1 / 4+\pi / 40$. As in Section 4 , we compare the orders of accuracy $p=2,4$, and 6 . The number of moment and smoothness conditions is set to $p$. The semi-discrete equation (39) is integrated in time by the explicit RK-4 method. We use the time step $\Delta t=\widetilde{h} \min J / 2$ for the 2 nd and 4 th order cases, and $\Delta t=(\widetilde{h} \min J)^{3 / 2} / 2$ for the 6 th order case. The source time function $g(t)$, the number of grid refinements, and the number of grid points are the same as in Section 4. Figure 9 shows the $l_{2}$-norm of the error in the numerical solution at the final time $T=20$. In all cases the error converges to zero at the expected rates.

\subsection{Source discretization near physical boundaries}

A stable discretization of the 1-D advection equation (1) with non-periodic boundary conditions can be constructed by using summation by parts (SBP) boundary closures of the difference operator $D$, see e.g. $[24,15]$. Here we focus on a diagonal norm SBP operator $\widetilde{D}$ whose order of accuracy is six in the interior of the domain and three in the boundary closure, which should result in a 4th order accurate numerical solution of the advection equation when the data is smooth. The operator satisfies the SBP relation

$$
(u, \widetilde{D} v)_{h, w}=-(\widetilde{D} u, v)_{h, w}-u_{0} v_{0}+u_{N} v_{N}
$$

in the weighted scalar product

$$
(u, v)_{h, w}=h \sum_{j=0}^{N} w_{j} \bar{u}_{j} v_{j}, \quad\|v\|_{h, w}=\sqrt{(v, v)_{h, w}} .
$$




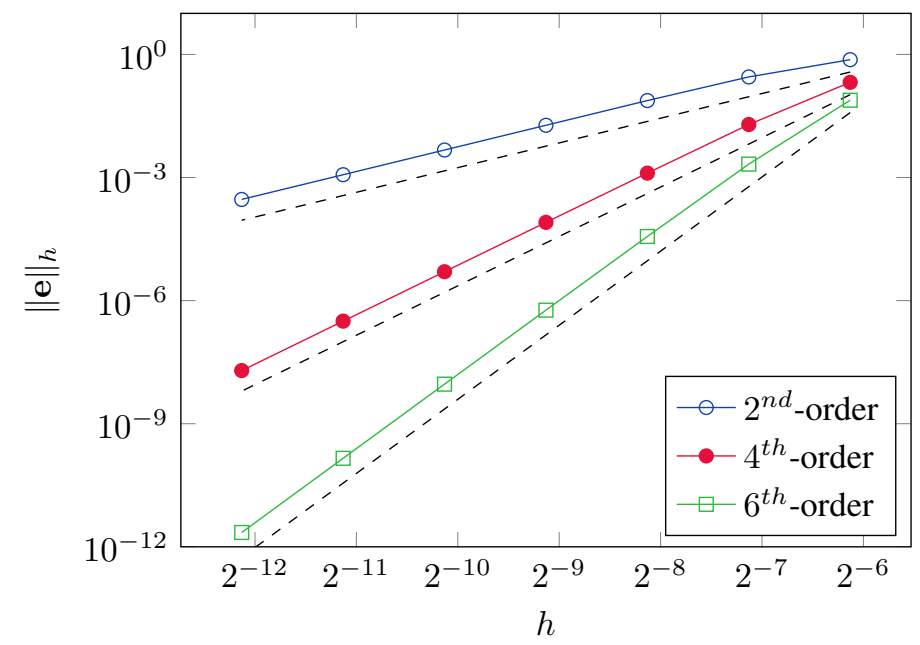

Figure 9: Error vs. grid size $h$ for $p=2,4$, and 6 with a curvilinear coordinate transformation. The source discretization satisfies $M=p$ moment conditions and $S=p$ smoothness conditions. The dashed lines show $\|e\|_{h} \sim h^{q}$, for $q=2,4,6$, respectively.

The weights are real and positive at all points. In particular, $w_{j}=1$ for $6 \leq j \leq N-6$, see [24] for details. Corresponding to (4), the semi-discrete problem becomes

$$
\begin{aligned}
\frac{d}{d t} v_{j}(t) & =-\widetilde{D} v_{j}+g(t) d_{j}, \quad j=0,1, \ldots, N, \quad t>0, \\
v_{j}(0) & =0 .
\end{aligned}
$$

We enforce the boundary condition $u(0, t)=0$ at the inflow boundary through the projection method [15], which in this case amounts to setting $v_{0}(t)=0$ throughout the time-stepping procedure. No boundary condition is needed at the outflow boundary and the summation by parts operator is applied all the way to the last grid point $x_{N}=L$. It is straightforward to derive a discrete energy estimate for $\|v\|_{h, w}$, showing that the approximation is stable. The boundary condition at $x=0$ could equally well have been enforced by a penalty term [6], but the additional modifications to the matrix $\widetilde{D}$ are not considered here. In what follows, $\widetilde{D}$ is the standard $S B P$ operator that satisfies (42).

For the wave equation in second order formulation, we have previously generalized the ideas of Waldén [27] to construct source discretizations near physical boundaries by enforcing the moment conditions (11) in a weighted scalar product such as (43). The resulting source discretization stencil becomes skewed near the boundary, but results in accurate numerical solutions $[19,20]$. However, as we have seen for the periodic case, a source discretization for the advection equation also needs to satisfy smoothness conditions. It turns out that enforcing the smoothness conditions (14) when the source is near the boundary leads to very inaccurate numerical solutions that sometimes even are inconsistent. The accuracy problem persists whether the smoothness conditions are enforced in the weighted or unweighted scalar product.

To understand how to generalize the smoothness conditions to bounded domains, we make an argument similar to the periodic case and let $\psi$ be a spurious numerical mode, 
i.e., a function that is not resolved on the grid. Similar to (12), we consider the weighted scalar product between $\psi$ and (44),

$$
\frac{d}{d t}(\psi, v)_{h, w}=-(\psi, \widetilde{D} v)_{h, w}+g(t)(\psi, d)_{h, w},
$$

which governs the evolution of the spurious component $(\psi, v)_{h, w}$. If $(\psi, \widetilde{D} v)_{h, w}=0$, we can only prevent $(\psi, v)_{h, w}$ from growing in time by enforcing the smoothness condition $(\psi, d)_{h, w}=0$. To derive an equation for $\psi$, we consider

$$
(\psi, \widetilde{D} v)_{h, w}=h \psi^{T} W \widetilde{D} v=h\left(\widetilde{D}^{T} W \psi\right)^{T} v, \quad W=\operatorname{diag}\left(w_{0}, w_{1}, \ldots, w_{N}\right),
$$

where $\widetilde{D}^{T}$ is the transpose of the operator $\widetilde{D}$ written in matrix form. Thus, $(\psi, \widetilde{D} v)_{h, w}=$ 0 implies $\widetilde{D}^{T} W \psi=0$. In other words, $\widetilde{\psi}=W \psi$ is a null vector of $\widetilde{D}^{T}$, and the smoothness condition for the source discretization becomes

$$
\left(W^{-1} \widetilde{\psi}, d\right)_{h, w}=0, \quad \widetilde{D}^{T} \widetilde{\psi}=0 .
$$

In matrix form, the difference operator $\widetilde{D}^{T}$ has a very large condition number and it turns out to be difficult to directly calculate its null vector in a numerically stable way. Instead we first calculate the singular value decomposition

$$
\widetilde{D}=U \Sigma V^{T}
$$

where the matrices $U$ and $V$ are orthonormal and $\Sigma$ is diagonal. From here, $\widetilde{D}^{T}=V \Sigma U^{T}$. If $\mathbf{u}^{\{k\}}$ is a column vector of the matrix $U$ then $\widetilde{D}^{T} \mathbf{u}^{\{k\}}=\sigma_{k} V \mathbf{e}_{k}$, which is equal to zero if the singular value $\sigma_{k}=0$. Here, $\mathbf{e}_{k}$ is a column vector with a one in position $k$ and zero elsewhere. This calculation shows that the null vector $\widetilde{\psi}$ can be obtained as the left singular vector of $\widetilde{D}$ corresponding to a zero singular value. We have numerically calculated the singular value decomposition of $\widetilde{D}$ on a finite grid with between 50-100 points. In all cases, one singular value is very close to zero and many orders of magnitude smaller than the other singular values. The corresponding left singular vector, shown on the top row of Figure 10, quickly tends towards $(-1)^{j}$ away from the end points. We think of this vector as a boundary modified version of $\psi^{(0)}$. It will be used to enforce the lowest order smoothness condition for bounded domains.

For the corresponding problem without boundaries, the condition $D^{T} \psi=0$ is satisfied for $\psi_{j}^{(0)}=(-1)^{j}$ because $D$ is skew-symmetric and $D \psi^{(0)}=0$ for all consistent centered difference approximations. We also have $D \psi^{(1)}=c_{0} \psi^{(0)}$ for some constant $c_{0}$, and thus $(D)^{2} \psi^{(1)}=c_{0} D \psi^{(0)}=0$. Hence, $\psi^{(0)}$ and $\psi^{(1)}$ are null vectors of $(D)^{2}$. It therefore seems plausible that the linear equation $\left(\widetilde{D}^{T}\right)^{2} \widetilde{\psi}=0$ could have two linearly independent nontrivial solutions that behave as $\psi^{(0)}$ and $\psi^{(1)}$ away from the boundaries. This assumption is confirmed by numerically computing the singular value decomposition of $\widetilde{D}^{2}$. It has two singular values that are very close to zero. The corresponding left singular vectors are shown in the top two rows of Figure 10. The first vector is the same as before and the second vector is of the form $(-1)^{j}\left(x_{j}-c_{1}\right)$ away from the end points. The latter vector will be used as a boundary modified version of $\psi^{(1)}$ when enforcing the smoothness conditions. The procedure can be repeated for higher powers of the matrix $\widetilde{D}$. Figure 11 

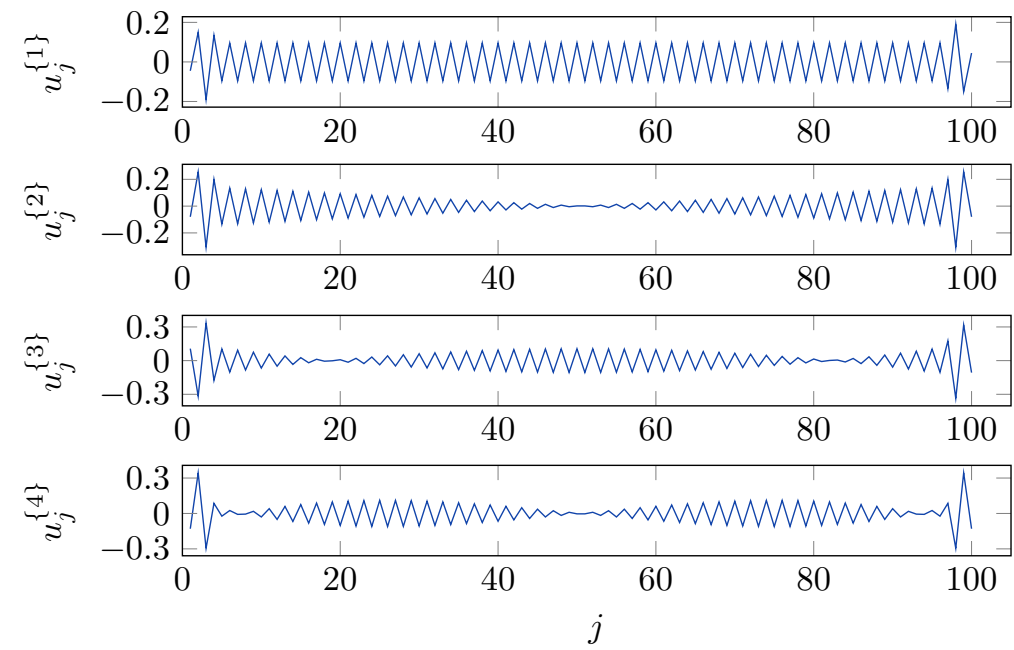

Figure 10: Left singular vectors corresponding to the four smallest singular values of the operator $(\widetilde{D})^{4}$ on a grid with 100 points.

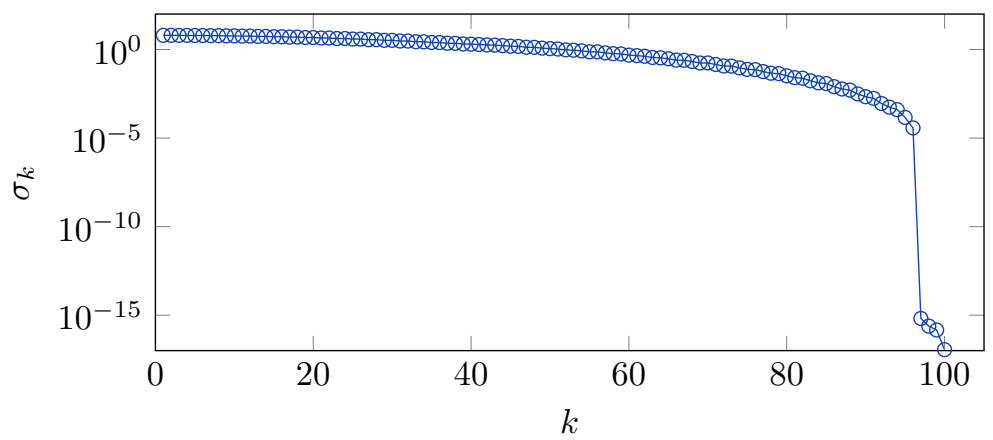

Figure 11: Singular values of the operator $(\widetilde{D})^{4}$ on a grid with 100 points.

shows numerically computed singular values of $(\widetilde{D})^{4}$ for a grid with 100 points. The last four singular values are very close to zero and at least 10 orders of magnitude smaller than the rest. The corresponding four left singular vectors are shown in Figure 10. Linear combinations of these vectors have been formed such that they behave as $(-1)^{j}$, $(-1)^{j}\left(x_{j}-c_{1}\right),(-1)^{j}\left(x_{j}-c_{2}\right)^{2}$, and $(-1)^{j}\left(x_{j}-c_{3}\right)^{3}$ in the interior of the domain, thereby showing the analogy with the periodic problem. Numerical calculations indicate that even higher powers of the difference operator, i.e., $(\widetilde{D})^{q}$ for $q \geq 5$, do not have more than four singular values that are close to zero. However, four smoothness conditions should be sufficient to achieve 4th order accuracy in the solution, which agrees with the design accuracy for the 6 th/3rd order accurate SBP operator under consideration here. SBP operators of other orders of accuracy might behave differently.

With four moment and four smoothness conditions, the source discretization stencil is always eight points wide. Similar to the periodic case, we define the center point of the 


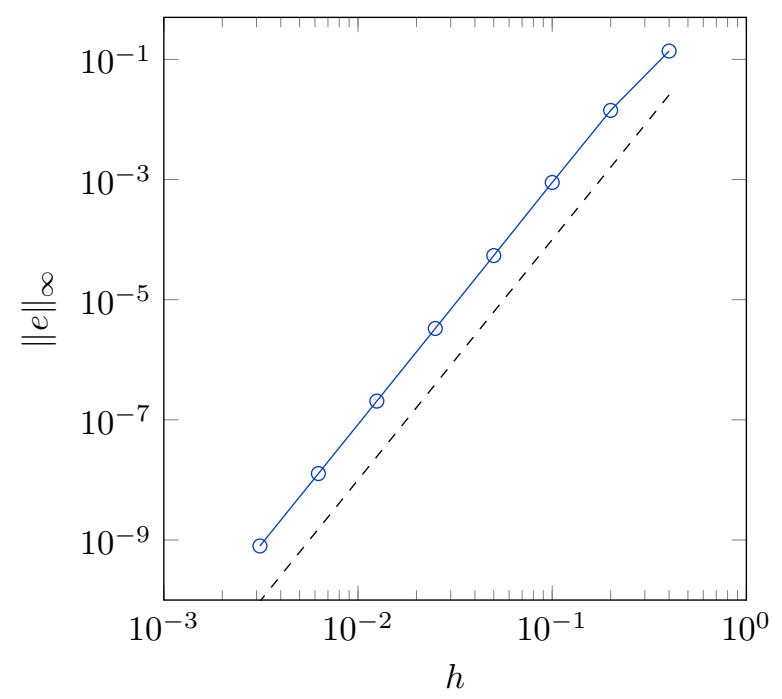

Figure 12: Maximum norm error vs. grid spacing when the source is on the boundary. Blue markers are errors from the computation. The black dashed line indicates 4 th order convergence rate.

stencil $k$ such that the source discretization $d_{j}$ is non-zero for $j \in[k-3, k+4]$. Because the projection method is used to enforce the boundary condition, the finite difference approximation is not applied at the boundary point itself. Therefore, $k \geq 4$ and we set $k=\max \left(4,\left\lfloor x_{*} / h\right\rfloor\right)$. As a result, $-4 \leq \alpha<0$ when $0 \leq x_{*}<x_{4}$.

In our implementation, we setup the source discretization on a bounded domain by first checking if $k>16$. In that case the moment conditions (11) and the smoothness conditions (14) are used without modifications, leading to the same formula as in the periodic case. If $k \leq 16$ the numerically computed null vectors replace $\psi^{(s)}$ in the smoothness conditions (14), and the moment conditions (11) are enforced in the weighted scalar product (43). To accommodate all possible locations of the eight point stencil, the first 20 elements of the numerically computed null vectors are stored in the solver.

We demonstrate the boundary source discretization by a numerical experiment where the advection equation (44) is solved on a domain of size $L=40$, where the source is placed directly on the boundary $\left(x_{*}=0\right)$, using the Gaussian source time function (35). Figure 12 shows the error in maximum norm at time $T=20$ vs. the grid size for a sequence of discretizations with increasingly finer grid size, clearly demonstrating that 4th order convergence is achieved. 4th order convergence has also been obtained for many other source locations near the boundary, but the numerical results are omitted to conserve space.

\section{Applications}

\subsection{The 3-D elastic wave equation}

To test the source discretization in 3 -D, it was implemented in the elastic wave propagation solver waveqlab3d [8]. This code employs a finite difference method that satisfies the principle of summation by parts $[12,24]$ for accurate simulations of seismic wave 
propagation. In this approach, the elastic wave equation is formulated as a system of nine first order hyperbolic partial differential equations,

$$
\begin{aligned}
& \rho \frac{\partial v_{k}}{\partial t}=\frac{\partial \sigma_{k 1}}{\partial x^{1}}+\frac{\partial \sigma_{k 2}}{\partial x^{2}}+\frac{\partial \sigma_{k 3}}{\partial x^{3}}, \\
& \frac{\partial \sigma_{k k}}{\partial t}=(\lambda+2 \mu) \frac{\partial v_{k}}{\partial x^{k}}+g(t) M_{k k} \delta\left(\mathbf{x}-\mathbf{x}_{*}\right), \quad k \in[1,3], \\
& \frac{\partial \sigma_{k j}}{\partial t}=\mu\left(\frac{\partial v_{k}}{\partial x^{j}}+\frac{\partial v_{j}}{\partial x^{k}}\right)+g(t) M_{k j} \delta\left(\mathbf{x}-\mathbf{x}_{*}\right), \quad j \in[k+1,3] .
\end{aligned}
$$

Here $\left(v_{1}, v_{2}, v_{3}\right)$ are the Cartesian components of the particle velocity and $\sigma_{k j}$ are the six unique components of the symmetric Cartesian stress tensor. The isotropic elastic medium is characterized by its density $(\rho)$ and the Lamé parameters $(\lambda$ and $\mu)$. The finite difference stencils used in our test problem are centered 6th order accurate in the interior and skewed third order accurate near the boundaries. Time stepping is handled by a low storage RK-4 scheme with a constant time step $\Delta t$, which is proportional to the grid size. This numerical method results in 4th order accuracy in space and time when the solution is sufficiently smooth [8].

Here we want to test the accuracy of waveqlab3d when the numerical solution is due to a point moment tensor source. This problem can be solved analytically for the case of an unbounded homogeneous isotropic elastic material, see for example Achenbach [2] (pp. 96-101).

The elastic body used in this test problem has the homogeneous material properties

$$
c_{p}=6000 \mathrm{~m} / \mathrm{s}, c_{s}=3464 \mathrm{~m} / \mathrm{s}, \rho=2670 \mathrm{~kg} / \mathrm{m}^{3},
$$

and the moment tensor source has components

$$
M_{k l}=10^{18} \mathrm{Nm}, \quad k, l=1,2,3 .
$$

We use a normalized Gaussian as source time function,

$$
g(t)=\frac{1}{\sigma \sqrt{2 \pi}} e^{-\left(t-t_{0}\right)^{2} / 2 \sigma^{2}}, \quad \int_{-\infty}^{\infty} g(\tau) d \tau=1,
$$

where $t_{0}$ is the center time and $\sigma$ is spread of the Gaussian, corresponding to its fundamental (angular) frequency $1 / \sigma$. In our test problem, we use $\sigma=0.1149 \mathrm{~s}$ and $t_{0}=0.7$ $\mathrm{s}$. This value of $t_{0}$ satisfies $t_{0}>6 \sigma$, which should ensure that the solution is free from numerical artifacts due to startup transients.

The computational domain $\mathbf{x} \in[-10000,10000]^{3} \mathrm{~m}$ is discretized on a uniform Cartesian grid, with constant grid size $h=100,50$, and $25 \mathrm{~m}$, respectively. Numerical far-field conditions are imposed on all boundaries, see [8] for details. The moment tensor source in our first test is located at $\mathbf{x}_{* 1}=(0,0,0) \mathrm{m}$, which coincides with a grid point for all grid sizes. In a second test, we place the source such that it lies between grid points for all of the above grid sizes, at $\mathbf{x}_{* 2}=(10,10,10) \mathrm{m}$. The three-dimensional source discretization is constructed as a Cartesian product of one-dimensional source discretizations in each coordinate direction. At the grid point $\mathbf{x}_{\mathbf{j}}=\left(x_{j_{1}}^{1}, x_{j_{2}}^{2}, x_{j_{3}}^{3}\right)$, the coefficients satisfy

$$
d_{\mathbf{j}}^{3 D}=\delta_{\varepsilon}\left(x_{j_{1}}^{1}-x_{*}^{1}\right) \delta_{\varepsilon}\left(x_{j_{2}}^{2}-x_{*}^{2}\right) \delta_{\varepsilon}\left(x_{j_{3}}^{3}-x_{*}^{3}\right),
$$



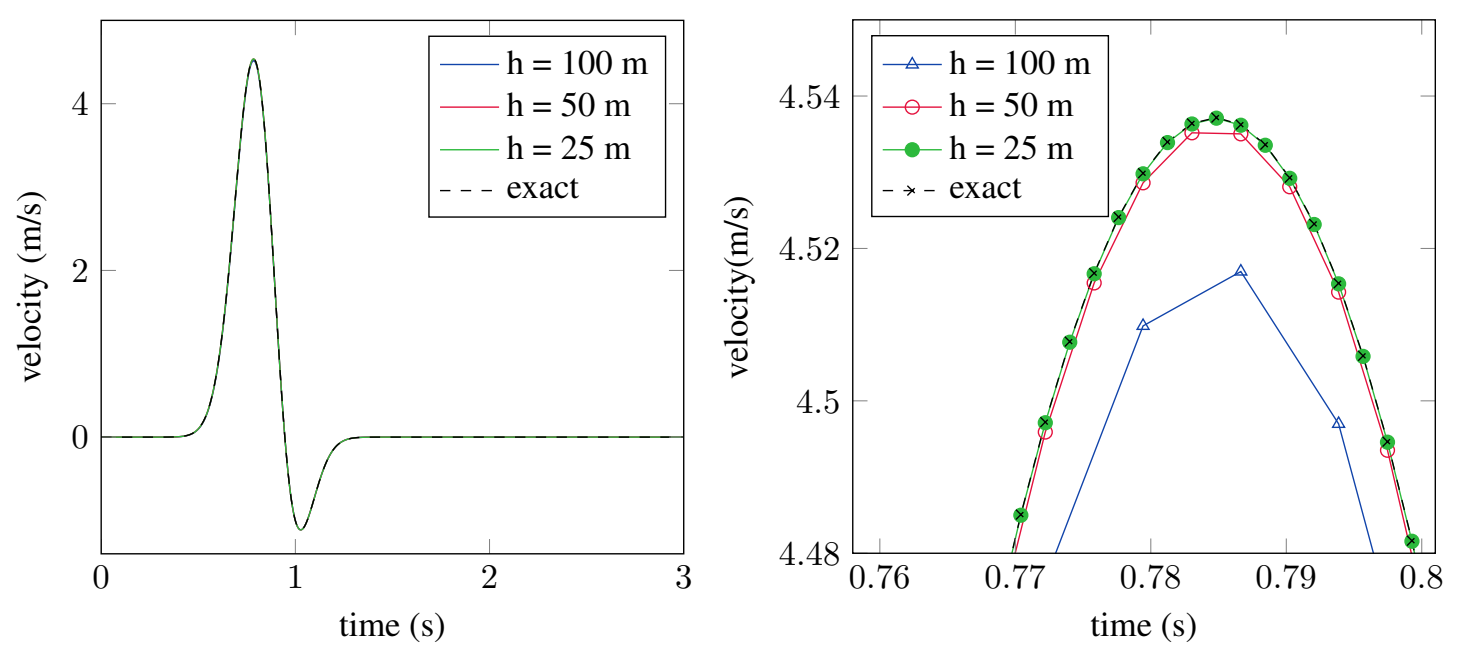

Figure 13: Numerical and exact $v_{1}$ component of the solution at $1 \mathrm{~km}$ distance from the source. A closeup near the first peak is shown on the right.

where $\mathbf{j}=\left(j_{1}, j_{2}, j_{3}\right)$ is a multi-index. In our case, the source is discretized by a 9 point stencil in each direction using $M=4$ moment conditions and $S=5$ smoothness conditions, forming a $9 \times 9 \times 9$ point cube around the source location over which the source discretization is distributed.

Figures 13-14 compare the analytical and numerical solutions as functions of time when the source is at $\mathbf{x}_{* 1}$. The comparison is made at two receivers, located at $\mathbf{x}_{r 1}=$ $(1000,0,0) \mathrm{m}$ and $\mathbf{x}_{r 5}=(5000,0,0) \mathrm{m}$, for the grid sizes 100,50 , and $25 \mathrm{~m}$. Figure 13 shows the radial $\left(v_{1}\right)$ component of the velocity at $\mathbf{x}_{r 1}$. Figure 14 shows one of the tangential components $\left(v_{2}\right)$ of the velocity at $\mathbf{x}_{r 5}$.

In Figure 15 we present the $L_{2}$ norm of the solution error for both source locations at receivers $\mathbf{x}_{r 1}$ and $\mathbf{x}_{r 5}$, respectively. The norm of the error is defined by

$$
e_{t}=\sqrt{\frac{1}{N_{t}} \sum\left[\left(v_{1}-v_{1}^{(e)}\right)^{2}+\left(v_{2}-v_{2}^{(e)}\right)^{2}+\left(v_{3}-v_{3}^{(e)}\right)^{2}\right]}
$$

where the sum is taken over $N_{t}$ time steps such that $N_{t} \Delta t=2.7063 \mathrm{~s}$ for each grid size. Here, $v_{1}, v_{2}, v_{3}$ are the components of the velocity obtained via numerical simulations using waveqlab3d and $v_{1}^{(e)}, v_{2}^{(e)}, v_{3}^{(e)}$ are the corresponding components of the analytical solution. These results indicate that the numerical solution converges towards the analytical solution as $\mathcal{O}\left(h^{4}\right)$, or better.

\subsection{3-D acoustic wave propagation}

Three-dimensional acoustic wave propagation can be modeled through the linearized Euler equations of compressible flow in an ideal gas. These equations can be formulated as a system of first order hyperbolic partial differential equations, which can be written on symmetric form as

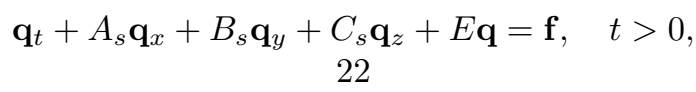



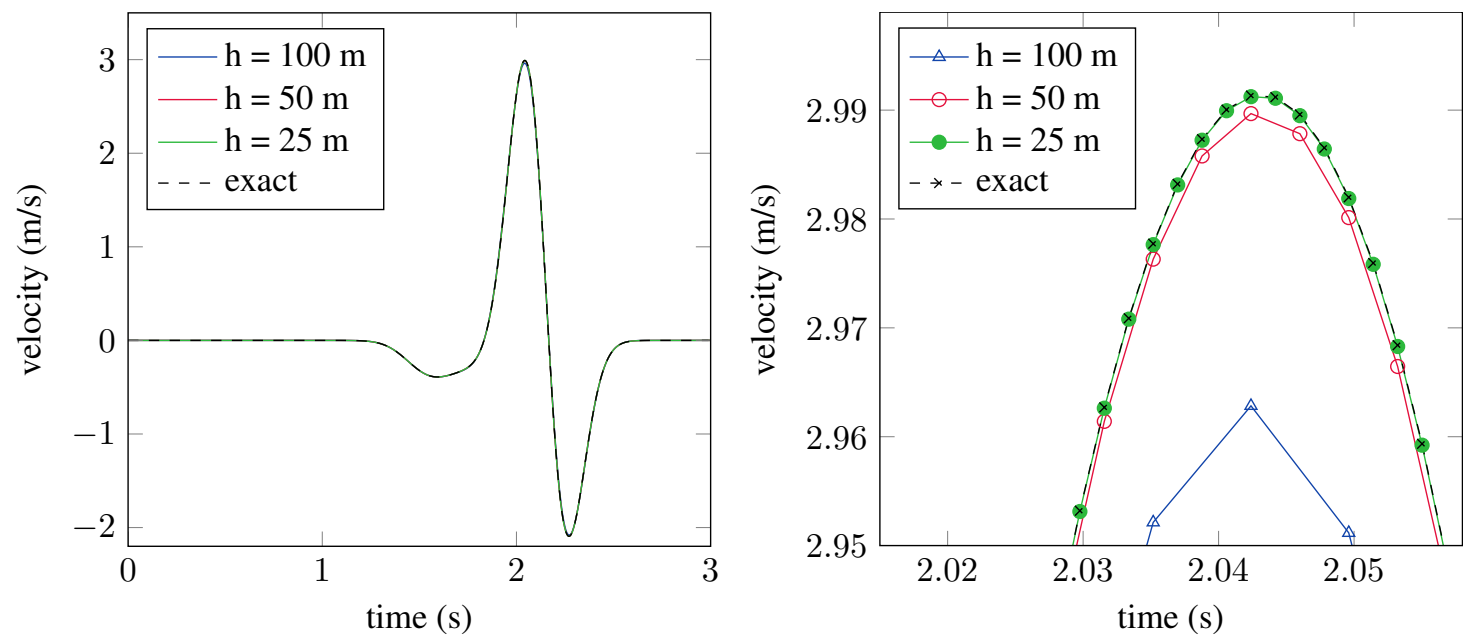

Figure 14: Numerical and exact $v_{2}$ component of the solution at $5 \mathrm{~km}$ distance from the source. A closeup of the peak near $t=2 \mathrm{~s}$ is shown on the right.
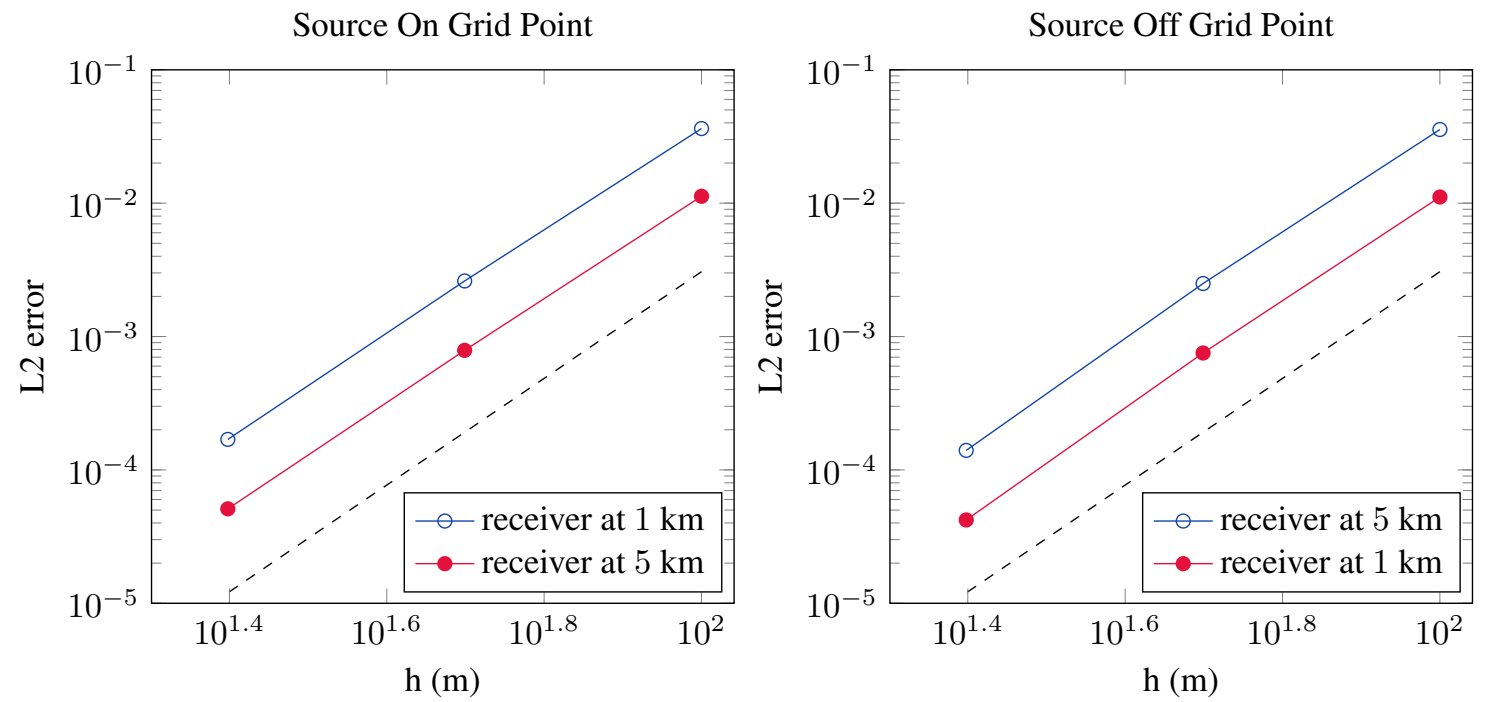

Figure 15: The $L_{2}$ error in the numerical solution of the elastic wave equation at the receiver locations $\mathbf{x}_{r 1}$ and $\mathbf{x}_{r 5}$, for grid sizes 100,50 , and $25 \mathrm{~m}$. The source is located on a grid point at $\mathbf{x}_{* 1}=(0,0,0)$ $m$ (left), and between grid points at $\mathbf{x}_{* 2}=(10,10,10) m$ (right). The dashed lines indicate $e_{t} \sim h^{4}$. 
subject to initial and boundary conditions. The dependent variable, $\mathbf{q}=\left(q_{1}, u, v, w, q_{5}\right)^{T}$, where

$$
q_{1}=\frac{\hat{c}}{\hat{\rho} \sqrt{\gamma}} \rho, \quad q_{5}=-\frac{\hat{c}}{\hat{\rho} \sqrt{\gamma(\gamma-1)}} \rho+\frac{\sqrt{\gamma}}{\hat{c} \hat{\rho} \sqrt{\gamma-1}} p,
$$

represent the acoustic perturbations around a background flow-field ${ }^{2}$, which may in general depend on $\mathbf{x}$ and $t$. The background flow-field has Cartesian velocity components $(\hat{u}, \hat{v}, \hat{w})^{T}$, pressure $\hat{p}$, and density $\hat{\rho}$. Furthermore, $q_{1}$ is a scaled density perturbation and $q_{5}$ is a linear combination of the perturbations of density and pressure. The speed of sound satisfies $\hat{c}=\sqrt{\gamma \hat{p} / \hat{\rho}}$, where $\gamma$ is a material constant that can be taken to be 1.4 in air.

The matrices $A_{s}, B_{s}$, and $C_{s}$ are symmetric and depend on the background flow field,

$$
A_{s}=\left(\begin{array}{ccccc}
\hat{u} & \frac{\hat{c}}{\sqrt{\gamma}} & 0 & 0 & 0 \\
\frac{\hat{c}}{\sqrt{\gamma}} & \hat{u} & 0 & 0 & \sqrt{\frac{\gamma-1}{\gamma}} \hat{c} \\
0 & 0 & \hat{u} & 0 & 0 \\
0 & 0 & 0 & \hat{u} & 0 \\
0 & \sqrt{\frac{\gamma-1}{\gamma}} \hat{c} & 0 & 0 & \hat{u}
\end{array}\right), \quad B_{s}=\left(\begin{array}{ccccc}
\hat{v} & 0 & \frac{\hat{c}}{\sqrt{\gamma}} & 0 & 0 \\
0 & \hat{v} & 0 & 0 & 0 \\
\frac{\hat{c}}{\sqrt{\gamma}} & 0 & \hat{v} & 0 & \sqrt{\frac{\gamma-1}{\gamma}} \hat{c} \\
0 & 0 & 0 & \hat{v} & 0 \\
0 & 0 & \sqrt{\frac{\gamma-1}{\gamma}} \hat{c} & 0 & \hat{v}
\end{array}\right)
$$

and

$$
C_{s}=\left(\begin{array}{ccccc}
\hat{w} & 0 & 0 & \frac{\hat{c}}{\sqrt{\gamma}} & 0 \\
0 & \hat{w} & 0 & 0 & 0 \\
0 & 0 & \hat{w} & 0 & 0 \\
\frac{\hat{c}}{\sqrt{\gamma}} & 0 & 0 & \hat{w} & \sqrt{\frac{\gamma-1}{\gamma}} \hat{c} \\
0 & 0 & 0 & \sqrt{\frac{\gamma-1}{\gamma}} \hat{c} & \hat{w}
\end{array}\right)
$$

The matrix $E$ depends on the gradient and time-derivative of the background flow field, and is zero when it is constant, i.e., independent of $\mathbf{x}$ and $t$. In the following we only consider the case $E=0$.

The term $\mathbf{f}$ on the right hand side of (49) models external forcing on the system. In general, the forcing can trigger both acoustic and non-acoustic waves. The two types of waves can, when the background flow field is constant and $\mathbf{f}=0$, be distinguished by making a plane wave ansatz for the solution of (49),

$$
\mathbf{q}(\mathbf{x}, t)=\mathbf{w} e^{i k(\mathbf{n} \cdot \mathbf{x}-c t)}, \quad \mathbf{n}=\left(n_{1}, n_{2}, n_{3}\right)^{T},|\mathbf{n}|=1 .
$$

Here, $k$ is the wave number, $\mathbf{n}$ is the direction of propagation and $c$ is the phase velocity. The ansatz leads to the eigenvalue problem $\widetilde{A} \mathbf{w}=c \mathbf{w}$, where $\widetilde{A}=n_{1} A_{s}+n_{2} B_{s}+n_{3} C_{s}$. The two acoustic waves correspond to the eigenvalues $c_{1,2}=\bar{u} \pm \hat{c}$, where $\hat{c}$ is the speed of sound and $\bar{u}=n_{1} \hat{u}+n_{2} \hat{v}+n_{3} \hat{w}$ is the speed of the background flow in the direction of the wave. There are also three non-acoustic waves, all propagating with phase velocity

\footnotetext{
${ }^{2}$ In this section, variables with a hat (e.g. $\hat{\rho}$ ) denote a background flow property and not a Fourier transformed variable.
} 
$c_{3,4,5}=\bar{u}$, corresponding to two transverse waves and one entropy wave. By forming a linear combination of the two acoustic eigenmodes, we arrive at a forcing term of the form

$$
\mathbf{f}(\mathbf{x}, t)=p_{0} g(t) \delta\left(\mathbf{x}-\mathbf{x}_{*}\right)\left(\mathbf{e}_{1}+\sqrt{\gamma-1} \mathbf{e}_{5}\right)
$$

which only triggers acoustic waves. Here, $\mathbf{e}_{1}$ and $\mathbf{e}_{5}$ are constant column vectors with zero elements, except for a unit element in the first and fifth row, respectively. Hence, the forcing is only applied to the first and fifth equation of (49).

An alternative way to interpret the forcing term (50) is to consider the special case of a constant background flow field with zero velocity, $\widehat{\mathbf{u}}=0$. Since $p=\widehat{\rho} \widehat{c}\left(\frac{1}{\sqrt{\gamma}} q_{1}+\frac{\sqrt{\gamma-1}}{\sqrt{\gamma}} q_{5}\right)$, and $\widehat{\rho} \mathbf{u}_{t}+\nabla p=0$, it is straightforward to derive a scalar wave equation for the pressure perturbation,

$$
p_{t t}=\hat{c}^{2} \nabla^{2} p+\widetilde{p}_{0} g^{\prime}(t) \delta\left(\mathbf{x}-\mathbf{x}_{*}\right), \quad \widetilde{p}_{0}=p_{0} \widehat{\rho} \widehat{c} \sqrt{\gamma} .
$$

The wave equation (51) is easily solved using spherical coordinates. In terms of $r=$ $\left|\mathbf{x}-\mathbf{x}_{*}\right|$ and retarded time $\tilde{t}=t-r / \hat{c}$, the solution satisfies

$$
p^{(e)}(r, \tilde{t})=\frac{\widetilde{p}_{0}}{4 \pi r \hat{c}^{2}} \begin{cases}0, & \tilde{t} \leq 0, \\ g^{\prime}(\tilde{t}), & \tilde{t}>0 .\end{cases}
$$

This expression will be used to evaluate the error in the numerical approximation of the full system (49), when the background flow field is constant and $\widehat{\mathbf{u}}=0$.

The linearized Euler equations (49) is discretized in space by a a 6 th order accurate finite difference method with third order accurate boundary modifications near physical boundaries [12, 24], and super-grid truncation of the computational domain at far-field boundaries [4, 20, 22]. The explicit 4th order accurate RK-4 scheme is used to integrate the solution in time. The method is implemented in the acoustic module of the code $E L A C$ [21].

The acoustic domain used in this test has homogeneous material properties

$$
\widehat{p}=1.025 \cdot 10^{5} \mathrm{~Pa}, \widehat{c}=331.02 \mathrm{~m} / \mathrm{s}, \widehat{\mathbf{u}}=0 \mathrm{~m} / \mathrm{s},
$$

and the point source has amplitude

$$
\widetilde{p}_{0}=10^{5} \mathrm{~Pa} \cdot \mathrm{m}^{3} .
$$

The source time function $g(t)$ is taken to be the normalized Gaussian (46). Here, we use the spread $\sigma=1 / 3.5 \mathrm{~s}$ and offset time $t_{0}=1.8 \mathrm{~s}$. As before, this value of $t_{0}$ satisfies $t_{0}>6 \sigma$, which should ensure that the solution is free from numerical artifacts due to startup transients.

The computational domain has the extent $(x, y) \in[0,4500] \mathrm{m}$ by $z \in[-4500,0] \mathrm{m}$. In our test, the system (49) is discretized on a Cartesian grid with uniform grid size $h$, for $h=30,15$, and $7.5 \mathrm{~m}$, respectively. Homogeneous initial conditions are imposed on all variables and the numerical solution is evolved until time $t=10 \mathrm{~s}$. The point source in our test is located at $\mathbf{x}_{*}=(2500,2000,-2500) \mathrm{m}$, which does not coincide with a grid point for any of the grid sizes. As in Section 5.1, the three-dimensional source discretization is formed as a Cartesian product of one-dimensional source discretizations 


\begin{tabular}{c|c|c|c}
\hline station & $x_{r}$ & $y_{r}$ & $z_{r}$ \\
\hline 1 & 2190 & 1980 & -2490 \\
\hline 2 & 1500 & 1980 & -2490 \\
\hline 3 & 1500 & 1980 & -1980 \\
\hline 4 & 1500 & 1980 & -1500 \\
\hline
\end{tabular}

Table 1: Cartesian coordinates of the receiver locations in the acoustic test.

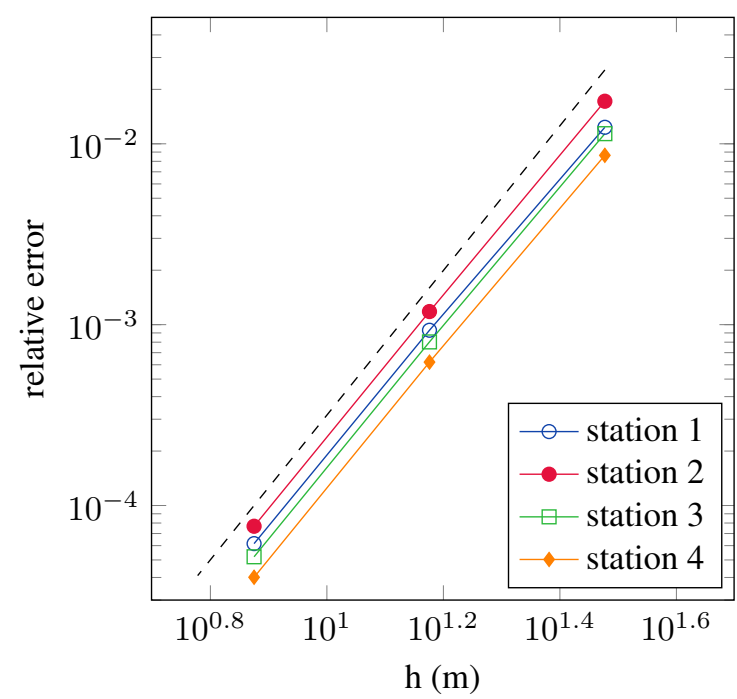

Figure 16: The relative error $\left(e_{r}\right)$ in the pressure in the numerical solution at the four receiver locations, for grid sizes 30, 15, and $7.5 \mathrm{~m}$, respectively. The dashed line indicates $e_{r} \sim h^{4}$.

according to (47). In each coordinate direction, the source is discretized by an 8-point stencil using $M=4$ moment conditions and $S=4$ smoothness conditions.

We test the accuracy of the numerical solution by evaluating it at the receiver locations in Table 1. Note that all receiver locations coincide with grid points for all of the grid sizes used in this test. In Figure 16 we present the relative errors in the pressure at the four receivers. The relative $L_{2}$ error is defined by

$$
e_{r}=\sqrt{\frac{\sum\left(p_{r}-p_{r}^{(e)}\right)^{2}}{\sum\left(p_{r}^{(e)}\right)^{2}}},
$$

where $p_{r}$ is the numerical solution at receiver $r, p_{r}^{(e)}$ is the analytical solution defined by (52), and the sums are evaluated over all time steps. These results indicate that the numerical solution is a 4 th order accurate approximation of the analytical solution. 


\section{Conclusions}

We have developed source discretizations for hyperbolic wave propagation problems in first order formulation that are discretized by high order accurate non-dissipative finite difference schemes in space, and integrated by explicit high order schemes in time, eg. Runge-Kutta methods. By studying the Fourier transform of the source discretization, we have derived sufficient conditions for achieving design accuracy in the numerical solution. Only half of the conditions in Fourier space can be satisfied through moment conditions on the source discretization, and we have introduced smoothness conditions for satisfying the remaining accuracy constraints. The resulting source discretization has compact support in physical space, and is distributed over as many grid points as the number of moment and smoothness conditions.

The theoretical properties of the source discretization have been verified numerically by solving the 1-D advection equation on a periodic domain using centered difference schemes of orders $p=2,4,6$. These experiments confirmed the theoretical prediction that the source discretization must satisfy $p$ moment and $p$ smoothness conditions to produce a $p$ th order accurate numerical solution. We generalized our technique to non-uniform grids using curvilinear coordinates. We also successfully tested the source discretization on two three-dimensional wave propagation problems, where the singular source was approximated by a Cartesian product of one-dimensional source discretizations.

For bounded domains we considered the advection equation discretized by a finite difference scheme that satisfies the principle of summation by parts. Here the moment conditions were enforced in a weighted scalar product and the smoothness conditions were generalized using numerically computed singular vectors of powers of the boundary modified difference operator. While this technique has proven to work well in numerical experiments, a more theoretical understanding would be desirable.

\section{Acknowledgments}

This work started while N.A. Petersson was on a sabbatical leave at the Institute for Computational and Mathematical Engineering (ICME) at Stanford University. Their hospitality is gratefully appreciated. This work performed under the auspices of the U.S. Department of Energy by Lawrence Livermore National Laboratory under contract DE-AC52-07NA27344. This is contribution LLNL-JRNL-679293.

\section{Appendix A. Centered difference operators}

The centered $2 \mathrm{nd}, 4 \mathrm{th}$, and 6 th order finite difference operators for approximating $\partial / \partial x$ are given by

$$
\begin{aligned}
& D^{(2)} v_{j}=\frac{1}{2 h}\left(v_{j+1}-v_{j-1}\right), \\
& D^{(4)} v_{j}=\frac{1}{12 h}\left(-v_{j+2}+8 v_{j+1}-8 v_{j-1}+v_{j-2}\right), \\
& D^{(6)} v_{j}=\frac{1}{60 h}\left(v_{j+3}-9 v_{j+2}+45 v_{j+1}-45 v_{j-1}+9 v_{j-2}-v_{j-3}\right) .
\end{aligned}
$$


Their Fourier symbols satisfy

$$
\begin{aligned}
\widehat{D}^{(2)}(\omega, h) & =\frac{i}{h} \sin (\omega h), \\
\widehat{D}^{(4)}(\omega, h) & =\frac{i}{h} \sin (\omega h)\left(1+\frac{2}{3} \sin ^{2}(\omega h / 2)\right), \\
\widehat{D}^{(6)}(\omega, h) & =\frac{i}{h} \sin (\omega h)\left(1+\frac{2}{3} \sin ^{2}(\omega h / 2)+\frac{8}{15} \sin ^{4}(\omega h / 2)\right),
\end{aligned}
$$

see [10] for details.

\section{Appendix B. Proof of Lemma 2}

Because $d_{j}=\mathcal{O}(1 / h)$, we have $\widetilde{d}_{j}:=h d_{j}=\mathcal{O}(1)$. We use the definition of the Fourier coefficients $(24)$ to define the continuous function $\widehat{d}(\omega h)$,

$$
\widehat{d}(\omega h):=\frac{1}{2 \pi} \sum_{j=0}^{N-1} \widetilde{d}_{j} e^{-i \omega h j},
$$

from which $\widehat{d}_{\omega}=\widehat{d}(\omega h)$. The moment conditions (11) can be written

$$
\begin{aligned}
(d, 1)_{h} & =1, \\
\left(d,\left(x-x_{*}\right)^{q}\right)_{h} & =0, \quad q=1, \ldots, p-1 .
\end{aligned}
$$

Let $x_{*}=(k+\alpha) h$, where $k$ is an integer and $0 \leq \alpha<1$, and expand the scalar products,

$$
\begin{aligned}
\sum_{j=0}^{N-1} \widetilde{d}_{j} & =1, \\
\sum_{j=0}^{N-1} \widetilde{d}_{j}(j-(k+\alpha))^{q} & =0, \quad q=1, \ldots, p-1 .
\end{aligned}
$$

We define the function $f(\omega h)$ by

$$
f(\omega h):=e^{i \omega x_{*}} \widehat{d}(\omega h)=e^{i \omega h(k+\alpha)} \widehat{d}(\omega h)=\frac{1}{2 \pi} \sum_{j=0}^{N-1} \widetilde{d}_{j} e^{i \omega h(-j+k+\alpha)} .
$$

Let $f^{(q)}$ denote the $q$ th derivative of $f$. Because of (B.3) and (B.4), it is straightforward to see

$$
\begin{aligned}
& f(0)=\frac{1}{2 \pi} \sum_{j=0}^{N-1} \widetilde{d}_{j}=\frac{1}{2 \pi}, \\
& f^{(q)}(0)=\frac{i^{q}}{2 \pi} \sum_{j=0}^{N-1} \widetilde{d}_{j}(-j+k+\alpha)^{q}=0, \quad q=1, \ldots, p-1 .
\end{aligned}
$$




\section{Taylor expansion gives}

$$
f(\omega h)=f(0)+\omega h f^{\prime}(0)+\ldots+\frac{(\omega h)^{p-1}}{(p-1) !} f^{(p-1)}(0)+\frac{(\omega h)^{p}}{p !} f^{(p)}\left(\xi_{1}\right),
$$

for some $0<\xi_{1}<\omega h$. Since the first $p-1$ derivatives of $f$ are zero at $\omega h=0, \widehat{d}=e^{-i \omega x_{*}} f$ satisfies

$$
\widehat{d}(\omega h)=\frac{1}{2 \pi} e^{-i \omega x_{*}}+\mathcal{O}\left((\omega h)^{p}\right),
$$

for $\omega h$ in a neighborhood of zero. This proves that (25) holds for some $0 \leq|\omega h| \leq \xi_{c}$, where $\xi_{c}>0$.

The smoothness conditions (14) can be written

$$
\sum_{j=0}^{N-1} \widetilde{d}_{j}(-1)^{j} j^{q}=0, \quad q=0, \ldots, p-1,
$$

where $0^{0}$ should be interpreted as 1 . It follows that

$$
\widehat{d}(\pi)=\frac{1}{2 \pi} \sum_{j=0}^{N-1} \widetilde{d}_{j} e^{-i \pi j}=\frac{1}{2 \pi} \sum_{j=0}^{N-1} \widetilde{d}_{j}(-1)^{j}=0,
$$

and

$$
\widehat{d}^{(q)}(\pi)=\frac{(-i)^{q}}{2 \pi} \sum_{j=0}^{N-1} \widetilde{d}_{j} j^{q}(-1)^{j}=0, \quad q=1, \ldots, p-1 .
$$

Taylor expansion around $\omega h=\pi$ gives

$$
\widehat{d}(\omega h)=\widehat{d}(\pi)+(\omega h-\pi) \widehat{d^{\prime}}(\pi)+\ldots+\frac{(\omega h-\pi)^{p-1}}{(p-1) !} \widehat{d}^{(p-1)}(\pi)+\frac{(\omega h-\pi)^{p}}{p !} \widehat{d}^{(p)}\left(\xi_{2}\right),
$$

where the remainder term is evaluated for some $\xi_{2} \in(\omega h, \pi)$. Since $\widehat{d}$ and its first $p-1$ derivatives are zero at $\omega h=\pi$, we conclude that

$$
|\widehat{d}(\omega h)|=\mathcal{O}\left(|\pi-\omega h|^{p}\right),
$$

for $\omega h$ in the vicinity of $\pi$. The same procedure applies for $\omega h$ near $-\pi$. For $|\omega h| \leq$ $\xi_{d}<\pi$, we use that $\widetilde{d}_{j}$ is only non-zero at some grid points $j=k_{1}, k_{1}+1, \ldots, k_{2}$, where $k_{2}-k_{1}+1=M+S$. Therefore,

$$
|\widehat{d}(\omega h)| \leq \frac{1}{2 \pi} \sum_{j=k_{1}}^{k_{2}}\left|e^{-\omega h j}\right|\left|\widetilde{d}_{j}\right| \leq \frac{M+S}{2 \pi} \max _{j}\left|\widetilde{d}_{j}\right| .
$$

Because the linear system (11)-(14) is non-singular, $\max _{j}\left|\widetilde{d}_{j}\right| \leq C$. Hence, there is a constant $c_{4}$ such that (26) is satisfied for all $|\omega h| \leq \pi$. 


\section{Appendix C. Proof of Lemma 3}

Inserting the Fourier coefficients of the solutions of the analytical (17) and semidiscrete (19) problems into (28) gives

$$
\widehat{e}_{\omega}(t)=\int_{0}^{t} g(\tau)\left(\frac{1}{2 \pi} e^{i \omega\left(\tau-t-x_{*}\right)}-e^{i \omega(\tau-t) P(\omega h)} \widehat{d}_{\omega}\right) d \tau
$$

We treat the four cases $\omega=0, h \leq|\omega h|<\xi_{c}, \xi_{c} \leq|\omega h|<\pi-\eta h^{\frac{m-1}{m}}$, and $\pi-\eta h^{\frac{m-1}{m}} \leq$ $|\omega h|<\pi$ separately.

The zeroth mode: $\omega=0$.. When $\omega=0$, Lemmas $1-2$ give $P=1$ and $\widehat{d}_{0}=1 / 2 \pi$, respectively. Therefore, $\widehat{e}_{0}(t)=0$, which proves the first case of Lemma 3 .

Low modes: $h \leq|\omega h|<\xi_{c}$. . By adding and subtracting $g(\tau) e^{i \omega(\tau-t)} \widehat{d}_{\omega}$ to the integrand, we can write (C.1) as a sum of two integrals,

$$
\begin{aligned}
\widehat{e}_{\omega}(t)=\int_{0}^{t} g(\tau) e^{i \omega(\tau-t)} & \left(\frac{1}{2 \pi} e^{-i \omega x_{*}}-\widehat{d}_{\omega}\right) d \tau \\
& +\int_{0}^{t} g(\tau)\left(e^{i \omega(\tau-t)}-e^{i \omega(\tau-t) P(\omega h)}\right) \widehat{d}_{\omega} d \tau=: T_{1}+T_{2}
\end{aligned}
$$

The idea is that $T_{1}$ is small because $\frac{1}{2 \pi} e^{-i \omega x_{*}}-\widehat{d}_{\omega}$ is small, and $T_{2}$ is small because $P$ is close to 1 , for small $|\omega h|$.

After repeated integration by parts,

$$
\begin{aligned}
T_{1}=e^{-i \omega t} \int_{0}^{t} g(\tau) e^{i \omega \tau} & \left(\frac{1}{2 \pi} e^{-i \omega x_{*}}-\widehat{d}_{\omega}\right) d \tau= \\
& -\frac{e^{-i \omega t}}{i \omega} \int_{0}^{t} g^{\prime}(\tau) e^{i \omega \tau}\left(\frac{1}{2 \pi} e^{-i \omega x_{*}}-\widehat{d}_{\omega}\right) d \tau= \\
\ldots & =(-1)^{p+1} \frac{e^{-i \omega t}}{(i \omega)^{p+1}} \int_{0}^{t} g^{(p+1)}(\tau) e^{i \omega \tau}\left(\frac{1}{2 \pi} e^{-i \omega x_{*}}-\widehat{d}_{\omega}\right) d \tau
\end{aligned}
$$

There are no boundary contributions because $g$ and its first $p+1$ derivatives are zero at $t=0$ and at $t>t_{1}$. Hence,

$$
\begin{aligned}
\left|T_{1}\right| \leq \frac{1}{|\omega|^{p+1}} \int_{0}^{t}\left|g^{(p+1)}(\tau)\right| & \left|\frac{1}{2 \pi} e^{-i \omega x_{*}}-\widehat{d}_{\omega}\right| d \tau \\
\leq & \frac{c_{3}|\omega h|^{p}}{|\omega|^{p+1}} \int_{0}^{t}\left|g^{(p+1)}(\tau)\right| d \tau \\
& =\frac{c_{3} h^{p}}{|\omega|} \int_{0}^{t}\left|g^{(p+1)}(\tau)\right| d \tau \leq \frac{C h^{p}}{|\omega|}
\end{aligned}
$$


and the desired estimate is proved for $T_{1}$. We estimate $T_{2}$ by integrating the second term of (C.2) by parts $p+2$ times and condense the notation by setting $P=P(\omega h)$,

$$
\begin{aligned}
T_{2}=(-1)^{p+2} & \int_{0}^{t} g^{(p+2)}(\tau)\left(\frac{1}{(i \omega)^{p+2}} e^{i \omega(\tau-t)}-\frac{1}{(i \omega P)^{p+2}} e^{i \omega(\tau-t) P}\right) \widehat{d}_{\omega} d \tau \\
= & \frac{1}{(-i \omega)^{p+2}} \int_{0}^{t} g^{(p+2)}(\tau) e^{i \omega(\tau-t)}\left(1-\frac{1}{P^{p+2}} e^{i \omega(\tau-t)(P-1)}\right) \widehat{d}_{\omega} d \tau
\end{aligned}
$$

Taking the absolute value gives

$$
\begin{aligned}
& \left|T_{2}\right| \leq \frac{1}{|\omega|^{p+2}} \int_{0}^{t}\left|g^{(p+2)}(\tau)\right|\left|1-\frac{1}{P^{p+2}} e^{i \omega(\tau-t)(P-1)}\right|\left|\widehat{d}_{\omega}\right| d \tau \\
& =\frac{1}{|\omega|^{p+2}} \int_{0}^{t}\left|g^{(p+2)}(\tau)\right| \frac{\left|P^{p+2}-1+1-e^{i \omega(\tau-t)(P-1)}\right|}{\left|P^{p+2}\right|}\left|\widehat{d}_{\omega}\right| d \tau
\end{aligned}
$$

By using (22) the upper bound $|P| \leq P_{\max }$, we can estimate

$$
\left|P^{p+2}-1\right|=|P-1|\left|P^{p+1}+P^{p}+\ldots+P+1\right| \leq c_{1}|\omega h|^{p} C .
$$

To analyze the second term, we note that

$$
\left|1-e^{i \alpha}\right| \leq|\alpha|,
$$

for any real $\alpha$. This and (22) give

$$
\left|1-e^{i \omega(\tau-t)(P-1)}\right| \leq|\omega(\tau-t)(P-1)| \leq|\omega t(P-1)| \leq c_{1}|\omega h|^{p}|\omega t| .
$$

Introducing these inequalities into (C.6) gives

$$
\begin{aligned}
\left|T_{2}\right| \leq \frac{c_{1}|\omega h|^{p}}{c_{5}^{p+2}|\omega|^{p+2}}(C+|\omega t|)\left|\widehat{d}_{\omega}\right| \int_{0}^{t}\left|g^{(p+2)}(\tau)\right| d \tau & \\
& \leq C h^{p}\left(\frac{1}{|\omega|^{2}}+\frac{t}{|\omega|}\right) \int_{0}^{t}\left|g^{(p+2)}(\tau)\right| d \tau
\end{aligned}
$$

where $c_{5}$ is a constant such that $|P(\omega h)| \geq c_{5}$ on $h \leq|\omega h| \leq \xi_{c}$. Note that (23) implies that such a bound can be found. Also, (26) implies that $\left|\widehat{d}_{\omega}\right| \leq C$. This proves that $\left|T_{2}\right| \leq C h^{p} /|\omega|$ for all fixed times $t>t_{1}$, since $1 /|\omega| \leq 1$ for $|\omega| \geq 1$.

High modes: $\xi_{c} \leq|\omega h|<\pi-\eta h^{\frac{m-1}{m}}$.. For the high modes, we treat the terms of (C.1) separately. Define

$$
T_{3}=\frac{1}{2 \pi} \int_{0}^{t} g(\tau) e^{i \omega\left(\tau-t-x_{*}\right)} d \tau,
$$

and

$$
T_{4}=-\int_{0}^{t} g(\tau) e^{i \omega(\tau-t) P(\omega h)} \widehat{d}_{\omega} d \tau
$$


so that $\widehat{e}_{\omega}=T_{3}+T_{4}$. By repeated integration by parts of (C.8),

$$
T_{3}=\frac{e^{-i \omega\left(t-x_{*}\right)}}{2 \pi(-i \omega)^{p+1}} \int_{0}^{t} g^{(p+1)}(\tau) e^{i \omega \tau} d \tau .
$$

It follows that

$$
\left|T_{3}\right| \leq \frac{1}{2 \pi|\omega|^{p+1}} \int_{0}^{t}\left|g^{(p+1)}(\tau)\right| d \tau .
$$

Because $|\omega h| \geq \xi_{c}$, we have $1 /|\omega| \leq h / \xi_{c}$, leading to the estimate

$$
\left|T_{3}\right| \leq \frac{h^{p}}{2 \pi \xi_{c}^{p}|\omega|} \int_{0}^{t}\left|g^{(p+1)}(\tau)\right| d \tau \leq \frac{C h^{p}}{|\omega|} .
$$

The estimate for $T_{4}$ is obtained by $p+m$ partial integrations of (C.9),

$$
T_{4}=\frac{1}{(-i \omega P)^{p+m}} \int_{0}^{t} g^{(p+m)}(\tau) e^{i \omega(\tau-t) P} \widehat{d}_{\omega} d \tau
$$

which gives, using (23) and (26),

$$
\begin{aligned}
\left|T_{4}\right| \leq \frac{c_{4}(\pi-|\omega h|)^{p}}{|\omega|^{p+m} c_{2}^{p+m}(\pi-|\omega h|)^{p+m}} & \int_{0}^{t}\left|g^{(p+m)}(\tau)\right| d \tau \\
& \leq \frac{c_{4}}{|\omega|^{p+m} c_{2}^{p+m}(\pi-|\omega h|)^{m}} \int_{0}^{t}\left|g^{(p+m)}(\tau)\right| d \tau .
\end{aligned}
$$

Because $|\omega h|<\pi-\eta h^{\frac{m-1}{m}}$,

$$
\frac{1}{\pi-|\omega h|}<\frac{1}{\eta h^{\frac{m-1}{m}}}
$$

and we obtain

$$
\left|T_{4}\right| \leq \frac{c_{4}}{c_{2}^{p+m} \eta^{m}|\omega|^{p}|\omega|^{m} h^{m-1}} \int_{0}^{t}\left|g^{(p+m)}(\tau)\right| d \tau \leq C \frac{h}{|\omega|^{p}} \leq C \frac{h^{p}}{|\omega|},
$$

where we used that $1 /|\omega h| \leq 1 / \xi_{c}$.

Remark 2. If the source discretization had satisfied $p+1$ instead of $p$ smoothness conditions, we could have obtained the estimate for $T_{4}$ using $p+1$ partial integrations. This estimate would be valid for $\xi_{c} \leq|\omega h| \leq \pi$, and eliminated the need to treat the very highest modes separately.

The highest modes: $\pi-\eta h^{\frac{m-1}{m}} \leq|\omega h|<\pi$.. The term $T_{3}$, defined by (C.8), can be estimated as above and the inequality (C.10) remains valid. Hence,

$$
\left|T_{3}\right| \leq C h^{p} /|\omega| \leq C h^{p+1} .
$$

After $p$ partial integrations of (C.9),

$$
T_{4}=\frac{1}{(-i \omega P)^{p}} \int_{0}^{t} g_{32}^{(p)}(\tau) e^{i \omega P(\tau-t)} \widehat{d}_{\omega} d \tau .
$$


It follows from (23) and (26) that

$$
\left|T_{4}\right| \leq \frac{c_{4}(\pi-|\omega h|)^{p}}{|\omega|^{p}|P|^{p}} \int_{0}^{t}\left|g^{(p)}(\tau)\right| d \tau \leq \frac{c_{4} h^{p}}{c_{2}^{p}|\omega h|^{p}} \int_{0}^{t}\left|g^{(p)}(\tau)\right| d \tau \leq C h^{p},
$$

since $1 /|\omega h| \leq 1 / \xi_{c}$. We conclude that $T_{3}+T_{4} \leq C h^{p}$. This completes the proof of Lemma 3.

[1] B. T. Aagard, R. Graves, A. Rodgers, T. M. Brocher, R. W. Simpson, D. Dreger, N. A. Petersson, S. C. Larsen, S. Ma, and R. C. Jachens. Ground motion modeling of Hayward fault scenario earthquakes, part II: Simulation of long-period and broadband ground motions. Bull. Seism. Soc. Amer., 100(6):2945-2977, 2010.

[2] J. D. Achenbach. Wave propagation in elastic solids, volume 16 of Applied Mathematics and Mechanics. North-Holland, 1973.

[3] K. Aki and P. G. Richards. Quantitative seismology. University Science Books, Sausalito, CA, USA, 2nd edition, 2002

[4] D. Appelö and T. Colonius. A high order super-grid-scale absorbing layer and its application to linear hyperbolic systems. J. Comput. Phys., 228:4200-4217, 2009.

[5] E. Audusse, F. Bouchut, M.-O. Bristeau, R. Klein, and B. Perthame. A fast and stable wellbalanced scheme with hydrostatic reconstruction for shallow water flows. SIAM J. Sci.Comput., $25: 2050-2065,2004$

[6] M.H. Carpenter, D. Gottlieb, and S. Abarbanel. Time-stable boundary conditions for finitedifference schemes solving hyperbolic systems: methodology and application to high-order compact schemes. J. Comput. Phys., 1993.

[7] C. de Groot-Hedlin, M. A. H. Hedlin, and K. Walker. Finite difference sythesis of infrasound propagation through a windy, viscous atmosphere: application to a bolide explosion detected by seismic networks. Geophys. J. Int., 185:305-320, 2011.

[8] K. Duru and E. M. Dunham. Dynamic earthquake rupture simulations on nonplanar faults embedded in 3D geometrically complex, heterogeneous elastic solids. J. Comput. Phys., pages -, 2015.

[9] L. Gosse. A well-balanced and asymptotic-preserving scheme for the one-dimensional linear Dirac equation. BIT, 55:433-458, 2015.

[10] B. Gustafsson, H.-O. Kreiss, and J. Oliger. Time dependent problems and difference methods. Wiley-Interscience, 1995.

[11] B. Hosseini, N. Nigham, and J. M. Stockie. On regularizations of the Dirac distribution. Technical report, Dept. of Math., Simon Frasier University, Canada, 8888 University Drive, Burnaby, BC, V5A 1S6, Canada, May 2015. arXiv:1412.4139v2.

[12] H.-O. Kreiss and G. Scherer. Finite element and finite difference methods for hyperbolic partial differential equations. In Mathematical aspects of Finite Elements in Partial differential equations. Academic Press, 1974

[13] M. J. Lighthill. On sound generated aerodynamically. Proc. Roy. Soc. Lon. A, 211(1107):564-587, 1952.

[14] Y. Mori. Convergence proof of the velocity field for a Stokes flow immersed boundary method. Comm. Pure Appl. Math., LXI:1213-1263, 2008.

[15] P. Olsson. Summation by parts, projections, and stability. I. Math. Comput., 64:1035-1065, 1995.

[16] S. Osher and J.A. Sethian. Fronts propagating with curvature dependent speed. J. Comput. Phys., 79:12-49, 1988.

[17] S. J. Osher and R. P. Fedkiw. Level set methods and dynamic implicit surfaces. Springer verlag, Berlin, 2002

[18] C. S. Peskin. The immersed boundary method. Acta Numerica, 11:479-517, 2002.

[19] N. A. Petersson and B. Sjögreen. Stable grid refinement and singular source discretization for seismic wave simulations. Comm. Comput. Phys., 8(5):1074-1110, November 2010.

[20] N. A. Petersson and B. Sjögreen. Super-grid modeling of the elastic wave equation in semi-bounded domains. Comm. Comput. Phys., 16:913-955, 2014.

[21] N. A. Petersson and B. Sjögreen. User's guide to ELAC, version 1.0. Technical Report LLNL-SMabcdef, Lawrence Livermore National Laboratory, 2015.

[22] N. A. Petersson and B. Sjögreen. Wave propagation in anisotropic elastic materials and curvilinear coordinates using a summation-by-parts finite difference method. J. Comput. Phys., 299:820-841, 2015 . 
[23] J. A. Sethian. Level set methods and fast marching methods. Evolving interfaces in computational geometry, fluid mechanics, computer vision and materials science. Cambridge university press, Cambridge, 1999.

[24] B. Strand. Summation by parts for finite difference approximations for d/dx. J. Comput. Phys., 110:47-67, 1994

[25] A.-K. Tornberg and B. Engquist. Numerical approximations of singular source terms in differential equations. J. Comput. Phys., 200:462-488, 2004.

[26] L. N. Trefethen. Group velocity in finite difference schemes. SIAM Rev., 24(2):113-136, 1982.

[27] J. Waldén. On the approximation of singular source terms in differential equations. Numer. Meth. Part. Diff. Eq., 15:503-520, 1999.

[28] W. Wang, C.-W. Shu, H.C. Yee, and B. Sjögreen. High order well-balanced schemes and applications to non-equilibrium flow. J. Comput. Phys., 228:6682-6702, 2009.

[29] W. Wang, C.-W. Shu, H.C. Yee, and B. Sjögreen. High order finite difference methods with subcell resolution for advection equations with stiff source terms. J. Comput. Phys., 231:190-214, 2012

[30] S. Zahedi and A.-K. Tornberg. Delta function approximations in level set methods by distance function extensions. J. Comput. Phys,, 229:2199-2219, 2010. 